\title{
The structure and origin of magnetic clouds in the solar wind
}

\author{
V. Bothmer ${ }^{1 *}$ and R. Schwenn ${ }^{2}$ \\ 1 Space Science Department of ESA, ESTEC, 2200 AG Noordwijk, The Netherlands \\ 2 Max-Planck-Institut für Aeronomie, 37189 Katlenburg-Lindau, Germany
}

Received: 29 January 1996 / Revised: 16 June 1997 / Accepted: 17 June 1997

\begin{abstract}
Plasma and magnetic field data from the Helios $1 / 2$ spacecraft have been used to investigate the structure of magnetic clouds (MCs) in the inner heliosphere. 46 MCs were identified in the Helios data for the period 1974-1981 between 0.3 and 1 AU. $85 \%$ of the MCs were associated with fast-forward interplanetary shock waves, supporting the close association between MCs and SMEs (solar mass ejections). Seven MCs were identified as direct consequences of Helios-directed SMEs, and the passage of MCs agreed with that of interplanetary plasma clouds (IPCs) identified as whitelight brightness enhancements in the Helios photometer data. The total (plasma and magnetic field) pressure in MCs was higher and the plasma- $\beta$ lower than in the surrounding solar wind. Minimum variance analysis (MVA) showed that MCs can best be described as largescale quasi-cylindrical magnetic flux tubes. The axes of the flux tubes usually had a small inclination to the ecliptic plane, with their azimuthal direction close to the east-west direction. The large-scale flux tube model for MCs was validated by the analysis of multi-spacecraft observations. MCs were observed over a range of up to $\sim 60^{\circ}$ in solar longitude in the ecliptic having the same magnetic configuration. The Helios observations further showed that over-expansion is a common feature of MCs. From a combined study of Helios, Voyager and IMP data we found that the radial diameter of MCs increases between 0.3 and $4.2 \mathrm{AU}$ proportional to the distance, $R$, from the Sun as $R^{0.8}$ ( $R$ in AU). The density decrease inside MCs was found to be proportional to $\mathrm{R}^{-2.4}$, thus being stronger compared to the average solar wind. Four different magnetic configurations, as expected from the flux-tube concept, for MCs have been observed in situ by the Helios probes. MCs with leftand right-handed magnetic helicity occurred with about equal frequencies during 1974-1981, but surprisingly, the majority (74\%) of the MCs had a south to north (SN) rotation of the magnetic field vector relative to the
\end{abstract}

*Present address: IFKKI, University of Kiel, 24118 Kiel, Germany Correspondence to: V. Bothmer ecliptic. In contrast, an investigation of solar wind data obtained near Earth's orbit during 1984-1991 showed a preference for NS-clouds. A direct correlation was found between MCs and large quiescent filament disappearances (disparition brusques, DBs). The magnetic configurations of the filaments, as inferred from the orientation of the prominence axis, the polarity of the overlying field lines and the hemispheric helicity pattern observed for filaments, agreed well with the in situ observed magnetic structure of the associated MCs. The results support the model of MCs as large-scale expanding quasi-cylindrical magnetic flux tubes in the solar wind, most likely caused by SMEs associated with eruptions of large quiescent filaments. We suggest that the hemispheric dependence of the magnetic helicity structure observed for solar filaments can explain the preferred orientation of MCs in interplanetary space as well as their solar cycle behavior. However, the whitelight features of SMEs and the measured volumes of their interplanetary counterparts suggest that MCs may not simply be just $\mathrm{H} \alpha$-prominences, but that SMEs likely convect large-scale coronal loops overlying the prominence axis out of the solar atmosphere.

\section{Introduction}

The term magnetic cloud (MC) was introduced by Burlaga et al. (1981) to characterize the magnetic field and plasma signatures of an interplanetary post-shock flow observed by five spacecraft separated over more than $30^{\circ}$ in solar longitude between 0.9 and $2 \mathrm{AU}$. The outstanding feature of this solar wind transient was the smooth rotation of the magnetic field vector nearly parallel to a plane over a time interval of the order of one day at $1 \mathrm{AU}$. This peculiar transient flow showed extremely low plasma- $\beta$ values, i.e., the plasma's magnetic pressure $\left(B^{2} / 8 \pi\right)$ dominated the thermal pressure $(n k T)$ due to its higher than average solar wind magnetic 
field strength $(B)$ and its unusually low plasma temperature $(T)$ and density $(N)$. The MC maintained its characteristic magnetic structure during its outward propagation up to at least 2 AU, although it had considerably expanded in its radial direction.

Subsequent analyses of solar wind data from various spacecraft near Earth and in the outer heliosphere revealed that MCs are relatively common phenomena in the solar wind (Klein and Burlaga, 1982; Burlaga and Behannon, 1982; Burlaga, 1991). MCs are often, but not always associated with interplanetary shocks (Klein and Burlaga, 1982; Zhang and Burlaga, 1988). MCs that strike the Earth may cause intense geomagnetic storms (Wilson, 1987; Zhang and Burlaga, 1988; Tsurutani et al., 1992; Bothmer, 1993; Bothmer and Schwenn, 1995).

The search for the solar origin of MCs brought up a close association of MCs with solar mass ejections (SMEs) and erupting prominences (disappearing filaments) (Burlaga et al., 1982; Wilson and Hildner, 1984, 1986; Marubashi, 1986; Bothmer and Schwenn, 1994). Note, that in this study we use the more general term 'solar mass ejection' (SME), rather than 'coronal mass ejection' (CME), because the origin and evolution of transient events in the solar atmosphere is not yet known from current white-light coronagraph observations and in situ solar wind measurements. Further, evidence has been found that at times not only coronal but also chromospheric solar material is ejected into interplanetary space (Schwenn et al., 1980). Here we prefer to reserve the term SME for the solar event and we call the interplanetary counterparts of SMEs ejecta, interplanetary plasma clouds (IPCs), or magnetic clouds (MCs) if they exhibit a specific magnetic topology. According to previous studies MCs represent just a subset of all IPCs found in the solar wind (Gosling, 1990; Bothmer and Schwenn, 1996). Among all IPCs identified by bidirectional electron (BDE) flows, $\sim 1 / 3$ had a large coherent internal magnetic field rotation characteristic of a MC (Gosling, 1990, 1993). Bothmer and Schwenn (1996) found from correlated white-light and in situ solar wind measurements that $41 \%$ of the driver gases (ejecta, IPCs), caused by SMEs directed towards the Helios spacecraft, were MCs.

Goldstein (1983) first suggested that the specific magnetic properties of MCs might be explained through force-free, large-scale, cylindrical magnetic flux tubes with $\nabla \times B=\alpha B$, i.e. the electric currents are flowing everywhere parallel or antiparallel to the magnetic field lines. Maximum magnetic field strength should be observed at the center, i.e., on the axis of the flux tube under ideal force-free conditions. Burlaga (1988), Marubashi (1986) and Lepping et al. (1990) found a good agreement between the in situ observed magnetic field characteristics of MCs and self-consistent magnetohydrodynamic (MHD)- calculations based on the forcefree flux tube concept. In contrast to the model of cylindrical shaped clouds, Vandas and Fisher (1991) and Vandas et al. (1993) suggested that MCs might rather be explained in terms of spheroidal topologies, and Farrugia et al. (1995) found that the flux tube model better describes in in situ field structure of MCs than the spheromak model.

The bidirectional flows of supra-thermal electrons along magnetic field lines, as observed inside IPCs, support the assumption of closed field lines, in agreement with observations that these plasma and magnetic field entities in the solar wind stem from regions in the solar atmosphere where the magnetic field lines form closed loops (Gosling et al., 1973; Montgomery et al., 1974; Bame et al., 1981; Gosling et al., 1987). It has been proposed that the ejecta from SMEs evolve into largescale magnetic flux ropes that remain magnetically connected to the solar atmosphere (e.g. Gosling, 1990; Gosling et al., 1992). Observations of solar energetic particles (SEPs) associated with MCs and IPCs (Kahler and Reames, 1991; Bothmer et al., 1996b), of flare particles inside a MC (Farrugia et al., 1993) and of BDE-anisotropies (Phillips et al., 1992) are also in favor of this picture. However, BDEs could be present on closed magnetic field lines disconnected from the Sun as in plasmoids, or on closed field lines that are rooted with its footpoints in the solar corona as in magnetic flux ropes. Unfortunately, current spacecraft measurements do not allow us to infer the overall topology of MCs unambiguously.

MCs have hitherto been studied extensively only at near Earth-orbit and beyond. The Helios mission has provided us with solar wind data in the inner heliosphere between 0.3 and 1 AU over a time interval covering more than one solar activity cycle. The purpose of our study is to extend the investigations of MCs to this range of the inner heliosphere. A further scientific goal of the work is to clarify the origin of MCs at the Sun, especially to validate the probable association with erupting solar prominences (disappearing filaments).

\section{Identification of magnetic clouds in the Helios data}

For the study presented here we used hourly averaged plasma and magnetic field data of the instruments E1 \& E2 onboard the Helios probes. A detailed description of the instruments can be found in the papers by Rosenbauer et al. (1981), Schwenn et al. (1975) and Musmann et al. (1975). The complete set of interplanetary magnetic field (IMF) and plasma data of Helios 1 covers the time interval December 1974 through July 1981. Data of Helios 2 span the time-interval January 1976 through March 1980.

Only a small number of magnetic clouds have been studied previously in the inner heliosphere at distances closer to the Sun than 1 AU (e.g., Burlaga et al., 1982). Thus, we had to establish appropriate identification criteria applicable to identify MCs between 0.3 and 1 AU. According to Burlaga (1991), MCs can be identified at 1 AU through the following criteria: (1) the magnetic field direction rotates parallel to a plane through a large angle during a time-interval of the order of one day; (2) the magnetic field strength inside a MC is higher than in the average solar wind; and (3) the temperature inside a $\mathrm{MC}$ is lower than average. Note, 
that the rotation of the magnetic field direction can occur in any direction relative to the ecliptic. Previous $\mathrm{MC}$ observations at $1 \mathrm{AU}$ have shown that the criteria (2) and (3) imply low plasma- $\beta$ values for MCs (Klein and Burlaga, 1982). Each individual criterion might occur independently of a MC, e.g. in association with planar magnetic structures (PMS) (Nakagawa et al., 1989; Neugebauer et al., 1993), co-rotating interaction regions (CIRs) or sector boundaries (SBs) (Bothmer and Schwenn, 1992a; 1996). For a unique identification of a MC all three criteria have to be fulfilled simultaneously.

The enormous radial size of $\mathrm{MCs}(\sim 0.25 \mathrm{AU}$ at $1 \mathrm{AU}$ ) was first evidence for an expansion of MCs in the heliosphere (Klein and Burgla, 1982). The Voyager spacecraft indeed observed larger radial sizes for MCs in the outer heliosphere (Burlaga and Behannon, 1982). The sizes of MCs observed near Earth's orbit cannot be considered to be typical of those that might be observed closer to the Sun. Further, the spacecraft's trajectory through a MC influences the observed duration time and thus its apparent size. Due to these uncertainties we concentrated our search for MCs in the Helios data on solar wind events associated with the basic property of a $\mathrm{MC}$, i.e., a smooth coherent directional change of the magnetic field vector on time scales of several hours to days. These changes can be identified in the magnetic field components $B_{\mathrm{x}}, B_{\mathrm{y}}, B_{\mathrm{z}}$ which are the Cartesian components of the IMF in solar ecliptic coordinates (SEC), and in the latitudinal and longitudinal excursions of the magnetic field vector, i.e., by variations in the angles $\theta$ and $\varphi . B_{x}$ points from the spacecraft towards the Sun, $B_{\mathrm{y}}$ points in the ecliptic plane normal to $B_{\mathrm{x}}$ towards the East, and $B_{\mathrm{z}}$ points normal to the ecliptic, towards the ecliptic north pole. Accordingly, $\theta$ and $\varphi$ are the magnetic field's polar $\left(\theta=90^{\circ} \equiv \mathrm{N}, \theta=-90^{\circ} \equiv \mathrm{S}\right)$ and azimuthal $\left(\varphi=0^{\circ} \equiv\right.$ sunward direction, $\varphi=90^{\circ}$ $\left.\equiv \mathrm{E}, \varphi=270^{\circ} \equiv \mathrm{W}\right)$ angles.

Based on these criteria we investigated plots of the plasma and magnetic field data of the Helios spacecraft taking into account all events in which variations in the magnetic field direction occurred in $\theta$ or $\varphi$ and in the cartesian components over time intervals of several hours. We included events with irregular or weak field variations and variations at sector boundaries. All events were then investigated with the minimum variance analysis (MVA) (Sonnerup and Cahill, 1967) described in the Appendix to identify smooth rotations of the magnetic field vector on time scales of the order of several hours. The MVA-method can be accurately applied for directional changes of the magnetic field vector exceeding $\sim 30^{\circ}$ (Burlaga and Behannon, 1982). Thus MCs were identified by a smooth rotation of the field direction in the plane of maximum variance, if the plasma temperature was lower and the magnetic field strength higher than in the surrounding solar wind (see criteria 2 and 3), and finally the error criteria of the minimum variance method were satisfied $\left(\lambda_{2} / \lambda_{3} \geq 2 ; \lambda_{2}\right.$ and $\lambda_{3}$ correspond to the eigenvalues of the directions of intermediate and minimum variance, see Appendix).

Figure 1 shows an example of a MC in the Helios data for a radial distance of $0.4 \mathrm{AU}$ from the Sun. The
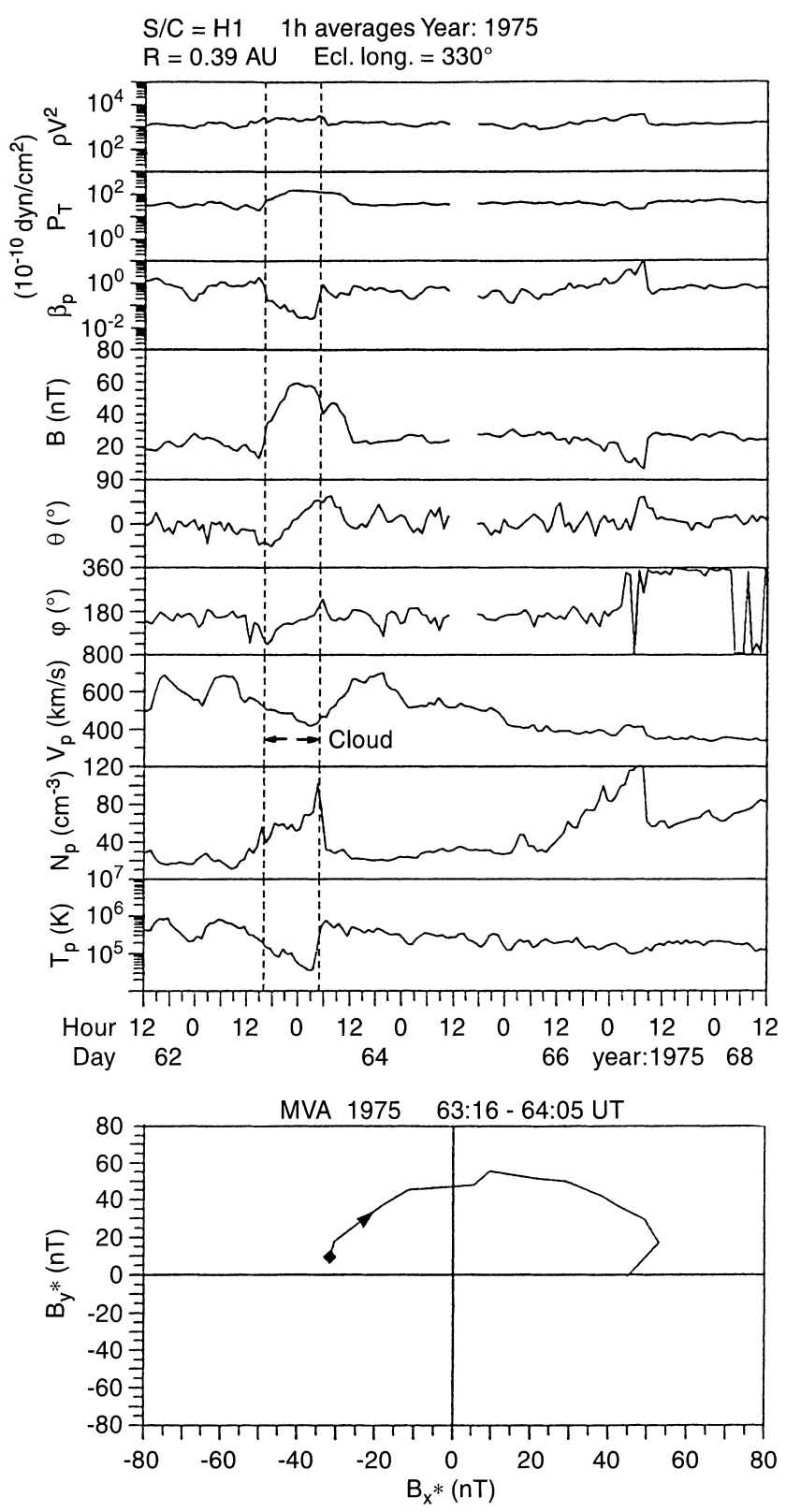

Fig. 1. Solar wind parameters for an MC (dashed lines) observed with Helios 1 at 0.4 AU in March 1975, day 63, 16 UT - day 64, 05 UT. Top to bottom: solar wind momentum flux $\rho_{p} V^{2}$, total plasma pressure $P_{T}\left(10^{-10} \mathrm{dyn} / \mathrm{cm}^{2}\right)$, plasma- $\beta_{p}$ calculated from the proton parameters, magnetic field magnitude $B[n T]$, polar and azimuthal angles $\theta, \varphi$ of the magnetic field vector in solar ecliptic coordinates, proton velocity $V_{p}$, proton number density $N_{p}$, and proton temperature $T_{p}$. The bottom part of the figure shows the rotation of the magnetic field vector in the plane of maximum variance for the time interval of the MC. The diamond denotes the initial point of the plot and the arrow indicates the direction of the rotation of the magnetic field

MC was basically identified from the smooth south to north turning of the magnetic field vector relative to the ecliptic. Note the rotation of $\theta$ in Fig. 1 covering the time interval marked with dashed lines, and that of the magnetic field vector in the plane of maximum variance. Here the diamond denotes the initial point of the plot and the arrow indicates the direction of the rotation of 
V. Bothmer, R. Schwenn: The structure and origin of magnetic clouds in the solar wind

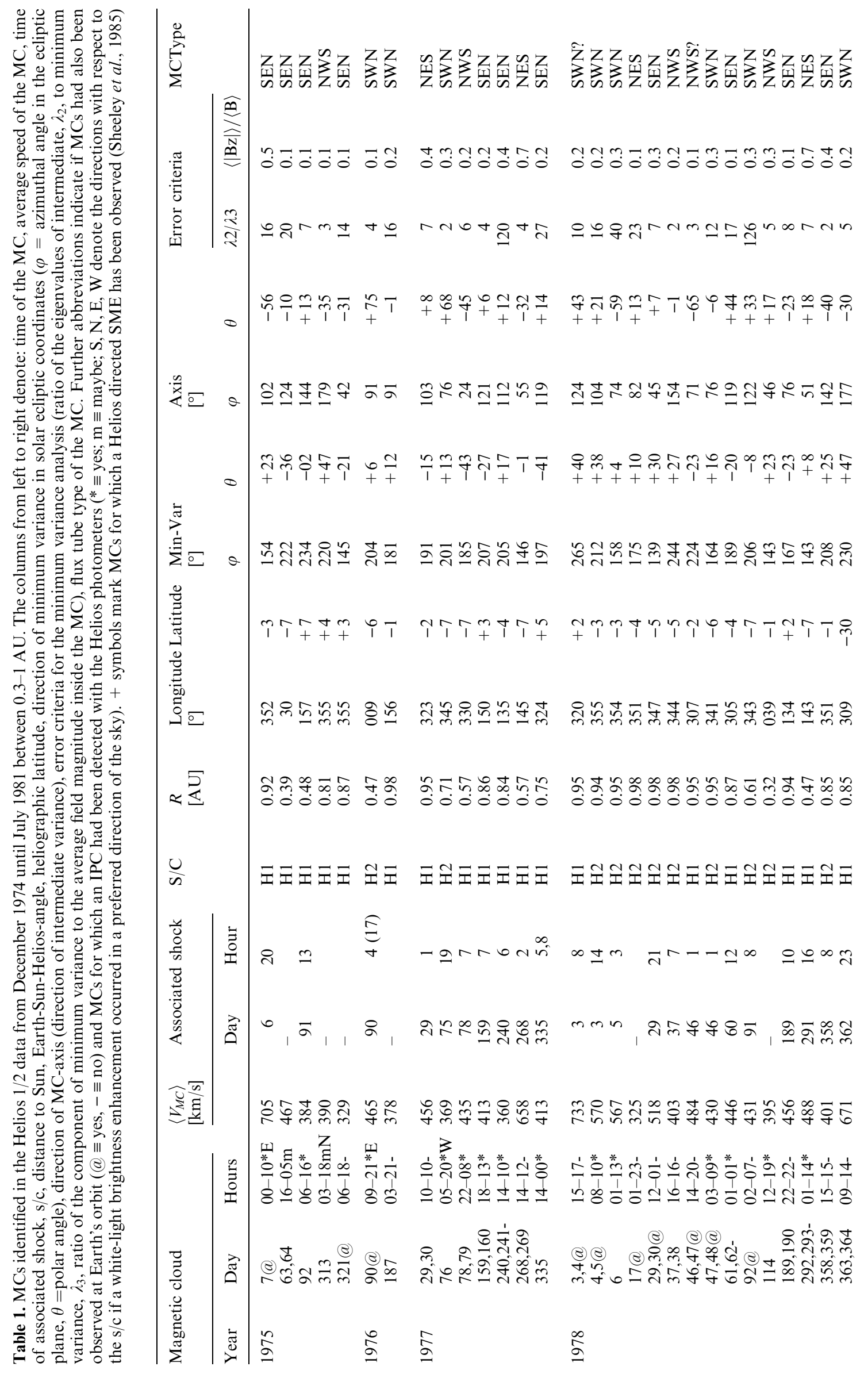




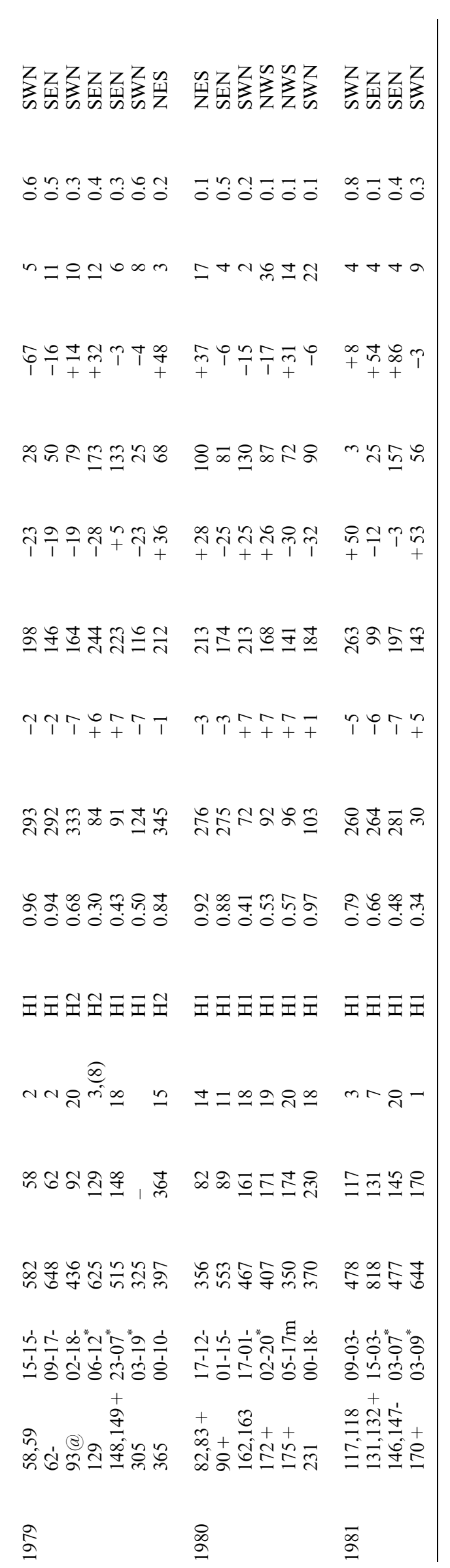

the magnetic field. The plasma- $\beta_{\mathrm{p}}$ (calculated from the proton parameters only) was much lower inside the cloud whereas the total plasma pressure $P_{T}\left(n k T+B^{2} / 8 \pi\right)$ was much higher compared with the surrounding flow. The solar wind momentum flux $\left(\rho V^{2}\right)$ remained fairly constant during the time interval shown in Fig. 1. Another interesting feature is the high plasma density compared with the ambient solar wind which may indicate that the low densities often found in MCs may be a result of considerable expansion of MCs with increasing distance from the Sun (see Klein and Burlaga, 1982; Burlaga and Behannon, 1982 and the results presented in Sec. 7).

Our search through the Helios data yielded a set of 46 certain MCs for the years 1974-1981 over the distance range 0.3-1 AU (Table 1). Note that many more cases hampered by data gaps or other disturbances were excluded. It is interesting to remark that generally solar wind structures with unique internal field rotations identified in the inner heliosphere were associated also with low plasma- $\beta$ values $(\beta \lesssim 0.1)$. This finding agrees with the characteristics of MCs at 1 AU (Burlaga, 1991).

\section{Magnetic clouds, interplanetary shocks and SMEs}

We examined the high resolution plasma and IMF data set of Helios $1 / 2$ to investigate the association of the identified MCs with interplanetary shock waves. 39 (85\%) of the $46 \mathrm{MCs}$ observed between $0.3-1 \mathrm{AU}$ were associated with fast-forward shocks (see Table 1). The arrival times of the MCs lagged the shocks by $5-25 \mathrm{~h}$ with an average delay of $\sim 11 \mathrm{~h}$. Solar wind momentum flux $\rho V^{2}$ often peaked near the "nose" of the MCs several hours after arrival of the interplanetary shock (e.g., Figs. 5, 6, 7). In agreement with theoretical models for interplanetary shocks (see, e.g., Hundhausen, 1972), MCs seem to be shock drivers in the inner heliosphere.

Figure 2 shows a histogram in $100 \mathrm{~km} / \mathrm{sec}$ bins for the average plasma speeds, $V$ (derived from the protons only), measured inside the $46 \mathrm{MCs}$ observed by Helios $1 / 2$. Most MCs were found to have a propagation speed similar to that of typically slow $(300-500 \mathrm{~km} / \mathrm{s})$ solar wind. Only a few MCs had speeds comparable to the typically high $(\sim 700-800 \mathrm{~km} / \mathrm{s})$ speed solar wind flow from coronal holes. The peak of the speed distribution in Fig. 2 lies in the range $400-500 \mathrm{~km} / \mathrm{s}$ with an average value of $\langle V\rangle=478(\mathrm{rms}=118) \mathrm{km} / \mathrm{s}$. As indicated in Fig. 2 by the open portion of the bars, the MCs that were not associated with shocks were amongst the slowest MCs with speeds typically less than $400 \mathrm{~km} / \mathrm{s}$. The finding that most of the MCs with speeds beyond $400 \mathrm{~km} / \mathrm{s}$ were associated with shocks is in agreement with the result obtained from direct correlations of coronagraph observations of SMEs directed towards Helios 1 and in situ shock disturbances at the spacecraft: When Helios 1 was located inside the angular extent of a SME seen in the coronagraph's field of view as viewed from Earth's orbit, it was definitely hit by an interplanetary shock as consequence of the SME, if the SME's speed was greater $400 \mathrm{~km} / \mathrm{s}$, independent of the space- 


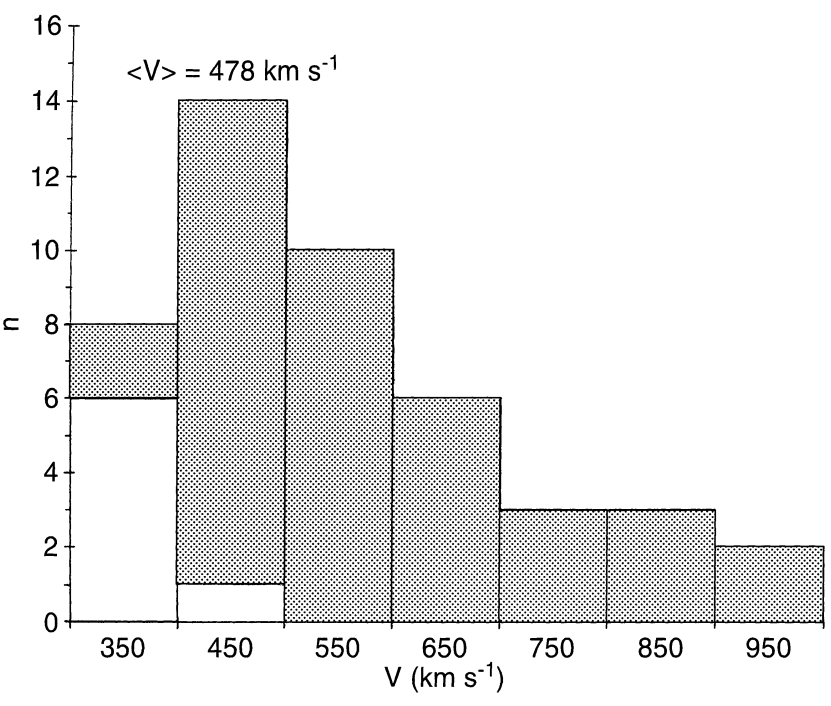

Fig. 2. Frequency distribution for the average proton speeds of MCs in bins of $100 \mathrm{~km} / \mathrm{s}$ as observed by Helios 1/2 during 1974-1981 between 0.3-1 AU. Open portions of the bars denote the number of MCs not associated with a shock. The average speed for MCs was $478 \mathrm{~km} / \mathrm{s}$

craft's distance to the Sun (Sheeley et al., 1985). The average speed of MCs shown in Fig. 2 is comparable to the speed distribution derived for SMEs (see Hundhausen et al., 1994) in the sense that the shape of the distribution drops off at speeds beyond $600 \mathrm{~km} / \mathrm{s}$, i.e., very fast MCs and SMEs are less frequent. However, the fact that the observed "low speed" MCs were so well associated with shocks may be indicative for deceleration of the MCs on their way out from the Sun depending on the ambient solar wind flow through which they had to plow. This should be especially important during solar maximum when the heliosphere consists of very inhomogeneous solar wind flow.

The high percentage of MCs associated with shocks found here is similar to that obtained for MCs observed near Earth orbit during the years 1978-1982 (Zhang and Burlaga, 1988) but higher than that observed during the years 1967-1978 (Klein and Burlaga, 1982). None of the 46 MCs observed between 0.3-1 AU was associated with a forward-reverse shock pair, which has been observed for interplanetary counterparts of SMEs detected by the Ulysses spacecraft at high heliographic latitudes in fast solar wind coronal hole flow (Gosling et al., 1994). Since it has been uniquely shown that fast SMEs at the Sun are the prime sources of transient shocks in the interplanetary medium (Sheeley et al., 1985; Schwenn, 1986; Cane et al., 1987) our findings support a close association of MCs with SMEs.

We compared our list of shock-associated MCs with the SME shock event list derived from correlated Helios-Solwind observations during 1979-1982 published by Sheeley et al. (1985) and found that 7 MCs occurred as direct consequences of SMEs towards Helios 1 (see Table 1). Another study based on this event list showed that 17 out of 25 Helios-directed SMEs which caused an interplanetary shock wave at Helios 1 were associated with driver gases, but only seven of the driver gases were MCs (Bothmer and Schwenn, 1996).
Note that this study included only those events of the original list of Sheeley et al. (1985) in which the presence or absence of a piston had been uniquely identified and for which both plasma and magnetic field measurements had been obtained.

Both observations are plausible since an interplanetary shock should commonly have a broader spatial extent than the associated driver gas (Borrini et al., 1982; Richardson and Cane, 1993) and IPCs might have different magnetic structures, as has been pointed out by Bothmer and Schwenn (1996).

The Helios photometers, designed to measure the zodiacal light, mounted at an angle of $16^{\circ}, 31^{\circ}$ and $90^{\circ}$ with respect to the spacecraft's spin-axis, have been used as wide-angle coronagraphs in order to identify SMEs by their white-light brightness increases close to the Sun and also as IPCs in the interplanetary medium (Webb and Jackson, 1990). Striking evidence was found that plasma clouds resulting from SMEs did pass the Helios spacecraft. We compared our list of MCs with the list of IPCs detected by the Helios photometers (Jackson et al., 1994). Without considering the duty-cycle of the instruments we find 23 of our MCs (see Table 1) associated with an interplanetary white-light event identified as an IPC, i.e., MCs are intimately connected to IPCs from SMEs (see also Webb et al., 1993). Brightness enhancements in the $16^{\circ}$ and $31^{\circ}$ photometers usually preceded the peak of the $90^{\circ}$ photometer by 1-2 days. The peak in the $90^{\circ}$ photometer data is thought to indicate the passage of a plasma cloud right at Helios. We find also that there were IPCs identified from the Helios photometer data that had not the structure of MCs, in agreement with the finding that MCs are only a subset of all IPCs (Gosling, 1990; Bothmer and Schwenn, 1996).

\section{Magnetic clouds as magnetic flux tubes}

Goldstein (1983) has proposed that the magnetic field characteristics of MCs could be explained through force-free large-scale cylindrical magnetic flux tubes. The idealized sketch in Fig. 3 depicts the magnetic field signatures expected for a cylindrical magnetic flux tube which moves radially away from the Sun, i.e., in the $\mathrm{x}$-direction, and centrally passes a Helios spacecraft in the ecliptic. Here, the maximum change in the magnetic field direction would occur normal to the ecliptic in the $B_{\mathrm{x}}^{*}$-component due to the south $(\mathrm{S})$ to north $(\mathrm{N})$ turning of the magnetic field vector. The component of minimum variance, $B_{\mathrm{z}}^{*}$, corresponds to the radial component $B_{\mathrm{x}}$, because $B_{\mathrm{x}}$ is zero in the whole MC. At the center of the flux tube, i.e., at the cloud's axis, the magnetic field is directed in the azimuthal $(+y-)$ direction [eastern $(E)$ direction]. This is also the direction of intermediate variance, because $B_{\mathrm{y}}^{*}$ is zero at the cloud's outer boundaries (on the surface of the cylinder). In reality, the flux-tube axis may have any orientation relative to the ecliptic plane and to the radial direction i.e., the actual variance directions $x^{*}, y^{*}, z^{*}$ usually differ from the solar ecliptic coordinate system in $x, y, z$. Whether the model is appropriate to describe the structure of 


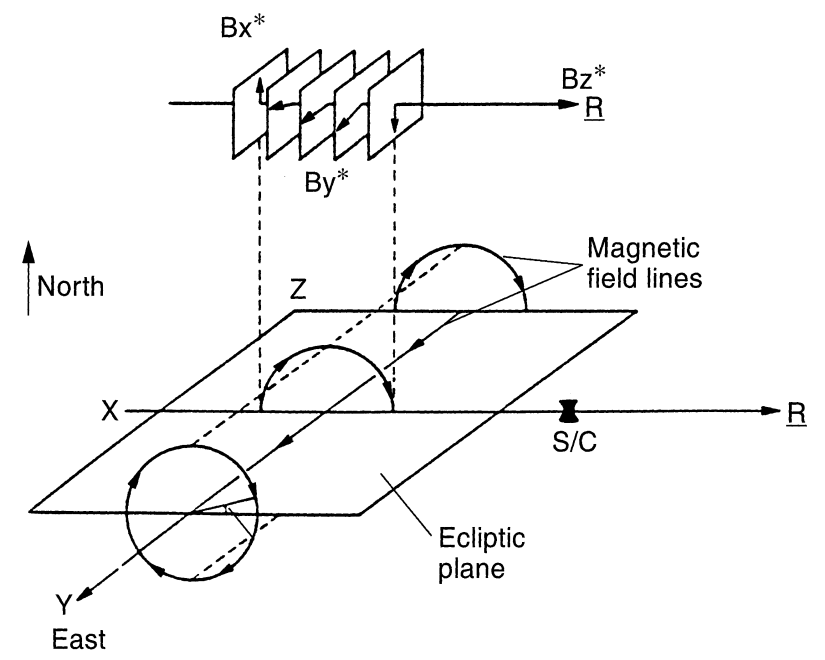

Fig. 3. Idealized sketch showing the expected magnetic field signatures during passage of a large-scale cylindrical magnetic flux tube over a $\mathrm{s} / \mathrm{c}$ (adapted from Goldstein, 1993). $B_{x}^{*}, B_{y}^{*}, B_{z}^{*}$ are the components of maximum, intermediate and minimum variance of the magnetic field vector. The south to north $\left[-B_{\mathrm{x}}^{*}(\mathrm{~S})\right.$ to $\left.+B_{\mathrm{x}}^{*}(\mathrm{~N})\right]$ turning of the magnetic field vector is associated with passage of the circular field lines at the cloud's outer boundaries. At the center of the flux tube (at the axis) the magnetic field is directed along the $+B_{\mathrm{y}}^{*}$ (east)-direction. A semicircular rotation appears when $B_{\mathrm{y}}^{*}$ is plotted versus $B_{\mathrm{x}}^{*}$ (top diagram)

MCs can be inferred from the results of MVA (Klein and Burlaga, 1982) applied to measured data.

In Fig. 4 the in situ characteristics of a MC associated with an interplanetary shock observed by Helios 1 are displayed together with the results of the variance analysis of the magnetic field data. A smooth rotation of the magnetic field direction from south to north (note the changes of $\theta$ in Fig. 4) starts in coincidence with a drop to very low $\beta_{\mathrm{p}}$ values ( $\beta_{\mathrm{p}}$ is calculated from the proton parameters only) at 01 UT on day 61. After the field vector has reached its most northern dip at 01 UT on day 62 it relaxes back to the average Parker spiral direction of the IMF. Note also the high total internal plasma pressure $P_{T}$ inside the MC. For shock-associated MCs we found the $P_{T}$-values usually to be highest between the shock and the leading edge of the cloud where the plasma turbulence is high. This region is commonly called the cloud's sheath (in analogy to the Earth's magnetosheath, see also McComas et al., 1988). Maximum pressure often occurred right at the nose of the MCs, thus indicating that MCs were indeed the drivers of the associated shocks. The large decrease of the magnetic field strength from the front to the rear portion of the cloud certainly stems from compression of the cloud's leading edge while plowing through slower moving ambient solar wind ahead. The in situ observations show that MCs are certainly not ideal force-free structures in reality.

The boundaries of the cloud have been determined from two parameters: the $\beta_{\mathrm{p}}$-slope and the time interval of rotation of the magnetic field vector. Note that unique criteria to determine MC-boundaries have not been established in the literature. The size of the MC as calculated from the cloud's average plasma speed and its
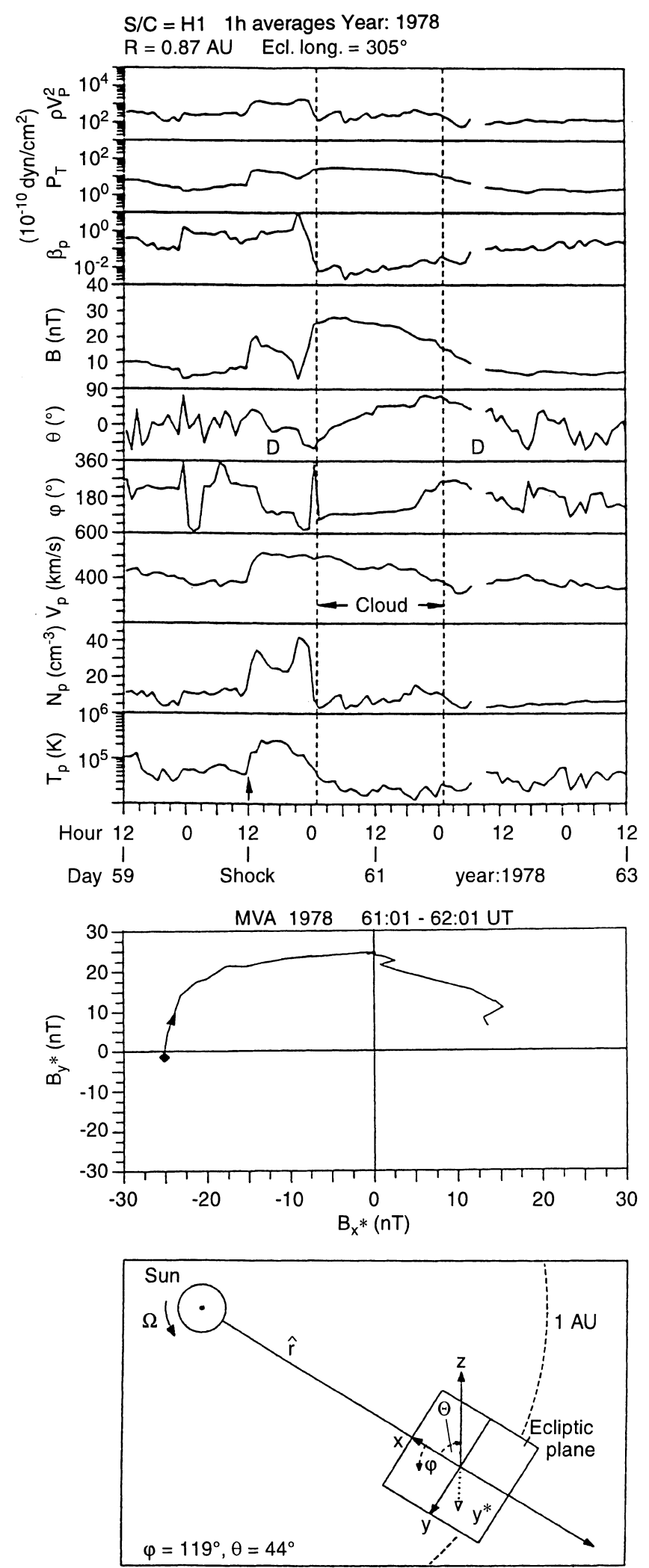

Fig. 4. Solar wind parameters (top panel) and results from the minimum variance analysis (middle panel) for a MC (dashed lines) observed with Helios 1 at 0.9 AU, day 61,01 UT - day 62,01 UT in 1978. The MC was associated with a shock on day 60, 12 UT. The draping of the IMF in the cloud's sheath region and behind the cloud's rear portion is labelled $(D)$. The calculated orientation of the cloud's axis, sketched in the bottom panel, was $\varphi=119^{\circ}, \theta=+44^{\circ}$. The direction of minimum variance was $\varphi=189^{\circ}, \theta=-20^{\circ}$ 
duration was $0.27 \mathrm{AU}$, a typical size for MCs observed at 1 AU (Klein and Burlaga, 1982).

Results of the MVA of the magnetic field data are presented in Fig. 4 in the lower diagrams. During the time interval of the $\mathrm{MC}, B_{\mathrm{z}}^{*}$ was small compared to the magnitude of the total magnetic field $B\left(\left\langle\left|B_{\mathrm{z}}^{*}\right|\right\rangle /\langle B\rangle\right.$ $=0.09)$. The ratio of the intermediate eigenvalue $\lambda_{2}$ to the minimum eigenvalue $\lambda_{3}$ was $\lambda_{2} / \lambda_{3}=16.8$, and the angle between the first and the last value of the field vector was $150^{\circ}$ (see Table 1 which includes the results of the MVA for all $46 \mathrm{MCs}$ ). The variance directions were well determined according to the error criteria given by Siscoe and Suey (1972) and Lepping and Behannon (1980). Plotted in the bottom panel in Fig. 4 is the component of intermediate variance $B_{\mathrm{y}}^{*}$ versus the component of maximum variance $B_{\mathrm{x}}^{*}$. The hodogram shows that the magnetic field vector rotates smoothly through a large angle in the plane of maximum variance.

The comparison of the in situ observed magnetic characteristics of this $\mathrm{MC}$ with that expected from the cylindrical flux tube model proposed by Goldstein (1983) is in excellent agreement. The calculated direction of the cloud's axis, i.e., the direction of intermediate variance, was $\varphi=119^{\circ}$ (azimuthal direction in the ecliptic), $\theta=+44^{\circ}$ (inclination relative to the ecliptic). In contrast to the in-ecliptic orientation of the flux tube axis sketched in Fig. 3, the axis of this MC had a considerable inclination relative to the ecliptic. A sketch of the axis direction at Helios 1 is given in Fig. 4 at the bottom. The calculated minimum variance direction was pointing southward of the ecliptic $\left(\theta=-20^{\circ}\right)$, slightly towards the west $\left(\varphi=189^{\circ}\right)$, close to the probable direction of propagation, which is radially outwards from the Sun.

Although more sophisticated cloud models have been developed, including also a possible offset of the spacecraft relative to the cloud's axis (Burlaga, 1988; Lepping et al., 1990; Vandas et al., 1993), a MVA of the magnetic field data is appropriate to estimate the cloud's axis orientation to first order.

\section{Configurations and orientations of MCs in interplanetary space}

The concept of cylindrical flux tubes allows different magnetic configurations. In the context of the investigation of the orientations of MCs in interplanetary space, it will be shown that all the predicted types of flux-tubes actually occur in the solar wind. Assume as in Fig. 3 that the axis of a MC lies in the ecliptic plane, normal to the sun-spacecraft line. A flux tube (MC) cloud possess one of the four different magnetic configurations presented in Table 2 , which differ in the orientation of the magnetic field lines at the cloud's outer boundaries and on its axes. These MCs would produce different characteristic magnetic signatures when passing over a spacecraft. According to Table 2, MCs can be classified into SEN (SWN) clouds where the magnetic field vector turns from south $(S)$ to east $(E)$ (west, W) on the cloud's axis and finally to the north (N) at its rear boundary, and vice versa into NES (NWS) clouds. More generally we can define $\Delta \theta>0^{\circ}$ for SNclouds $\left(\Delta \theta<0^{\circ}\right.$ for NS clouds) with $180^{\circ}<\varphi<0^{\circ}$ $\left(360^{\circ}>\theta>180^{\circ}\right)$ for SEN, NES (SWN, NWS) clouds. The classification introduced here involves the property of magnetic helicity (see Burlaga, 1988; Lepping et al., 1990). SEN and NWS clouds possess left-handed (LH), SWN and NES clouds, right-handed (RH) helicity as viewed by an observer looking towards the Sun. In situ observations of these four different MC-types will be presented in the following section.

\subsection{Magnetic cloud types}

5.1.1 SEN-type MC. Figure 5 shows a shock-associated SEN-cloud observed by Helios 1 in August 1977. The magnetic field direction changes smoothly from south to north during the cloud's passage over the spacecraft. Note that the southward dips of the magnetic field vector in the cloud's sheath region (see the variations of $\theta$, labelled D, in Fig. 5) were caused by draping of the IMF ahead of the MC (Gosling and McComas, 1987; McComas et al., 1988). At the cloud's center the magnetic field had an eastward direction. In the plane of maximum variance we find a rotation of the magnetic field vector from negative to positive $B_{\mathrm{x}}^{*}$-values and $+B_{\mathrm{y}}^{*}$ values. In this case, the component $B_{z}^{*}$ along the direction of minimum variance had a constant, but substantial non-zero value (see Table 1) which might be indicative of twisted rather than pure circular outer magnetic field lines. The ratio of $\lambda_{2} / \lambda_{3}$ was 120 , and the direction of minimum variance was $\varphi=205^{\circ}, \theta=+17^{\circ}$ in SECs. The cloud's axis was lying almost parallel to the ecliptic plane $\left(\theta=12^{\circ}\right)$, normal to the sun-spacecraft-line $\left(\varphi=112^{\circ}\right)$. Note also the high internal total plasma pressure $P_{T}$ and the very low plasma- $\beta_{p}$ inside the cloud. Other interesting features of this MC were its high magnetic field strength with a very symmetric time profile and the extreme drop of the plasma temperature near the center of the cloud.

5.1.2 SWN-type MC. A shock-associated SWN-cloud observed in December 1978 is presented in Fig. 6. The magnetic field changes of $\theta$ and $\varphi$ look relatively moderate, but a unique rotation of the field vector is present in the plane of maximum variance. Note that this cloud might have gone undetected had the data simply been inspected by eye. The axis of the cloud was lying close to the sun-spacecraft line $\left(\varphi=177^{\circ}\right)$, pointing slightly towards the south $\left(\theta=-30^{\circ}\right)$. The minimum variance direction was $\varphi=230^{\circ}, \theta=+47^{\circ}$. Inside the cloud $\beta_{p}$ is low and $P_{T}$ is high, as is typical. The strong decrease of the magnetic field strength from the cloud's front to its rear has probably been caused by interaction of this MC with the ambient solar wind.

5.1.3 NES-type MC. A shock-associated NES-cloud observed by Helios 1 in January 1977, directly at a 
Table 2. Sketch showing the different magnetic configurations of MCs and their magnetic helicity (left-handed (LH), right-handed (RH)) based on the magnetic fluxtube concept and the field rotation that a $\mathrm{s} / \mathrm{c}$ would observe during the cloud's passage. The number at the bottom indicates how often each MC-type was observed by Helios $1 / 2$ between $0.3-1$ AU during 1974-1981

\begin{tabular}{|l|l|l|l|}
\hline MC Type Magnetic helicity & $\begin{array}{c}\text { Variation of } \\
\text { magnetic field vector }\end{array}$ & $\begin{array}{c}\text { Direction of } \\
\text { magnetic field } \\
\text { on flux tube axis }\end{array}$ & $\begin{array}{c}\text { Rotation } \\
\text { of magnetic field } \\
\text { vector in } B \mathrm{z}-B \mathrm{y} \text {-plane } \\
\left(B \mathrm{x}^{*}-B \mathrm{y}^{*}-\text { plane }\right)\end{array}$ \\
\hline Number of MCs during 1974-1981
\end{tabular}

sector boundary (see the changes of $\varphi$ ) is shown in Fig. 7. The peak of the magnetic field strength at the cloud's front suggests strong interaction with the ambient plasma, which certainly also deformed the magnetic field lines. Although the field vector changes direction from $90^{\circ} \mathrm{N}$ to $0^{\circ}$, with $\varphi \approx 90^{\circ}$ at the cloud's center, the plane of maximum variance revealed a rather complex rotation, presumably a result of compression and distortion of the field lines due to the cloud's interaction with the ambient slower moving solar wind. An extremely high overabundance of $\mathrm{He}^{+}$has been measured inside this cloud (Schwenn et al., 1980) being most probably cold chromospheric material caused by an erupting prominence (Cane et al., 1986). The cloud's axis was lying in the ecliptic $\left(\theta=+8^{\circ}\right)$, normal to the sun-spacecraft line $\left(\varphi=103^{\circ}\right)$. The direction of minimum variance was near the ecliptic, approximately radially outwards $\left(\varphi=191^{\circ}, \theta=-15^{\circ}\right)$. Schwenn et al. (1980) explicitly stated that they did not find any evidence for the end-time of the driver gas within $48 \mathrm{~h}$ following the arrival of the shock wave at $\sim 01$ UT on January 29. However, the rotation of the magnetic field vector ends $15 \mathrm{~h}$ earlier, around $\sim 10$ UT on January 30 . In this case, the MC might have been the leading portion of a much larger plasma cloud from a SME, consistent with observations of intermittent intervals of BDEs following the MC (private communication, K. Ivory, Max-Planck-Institut für Aeronomie, Katlenburg-Lindau, Germany), as was the case for an IPC observed recently with the Ulysses spacecraft (Gosling et al., 1995a; Bothmer et al., 1996b). Events like these need further investigation to clarify their nature.

5.1.4 NWS-type MC. A shock-associated NWS-cloud passed Helios 1 in June 1980 (Fig. 8, see also Burlaga et al., 1982). It was directly associated with a Heliosdirected SME detected with the Solwind-coronagraph onboard the P78/1 satellite (Sheeley et al., 1985). The MC shows low $\beta_{p}$ and high $P_{T}$. The magnetic field vector turns from north $(\mathrm{N})$ over west $(\mathrm{W})$ finally to the south (S) at the cloud's rear. Note also the draping of the IMF in the sheath region between the shock and the MC, associated with high $\beta_{p}$ values, and the field variations following the cloud when the surrounding IMF drapes back to its undisturbed orientation. The cloud's axis was lying close to the EW-direction $\left(\varphi=87^{\circ}\right)$, slightly 

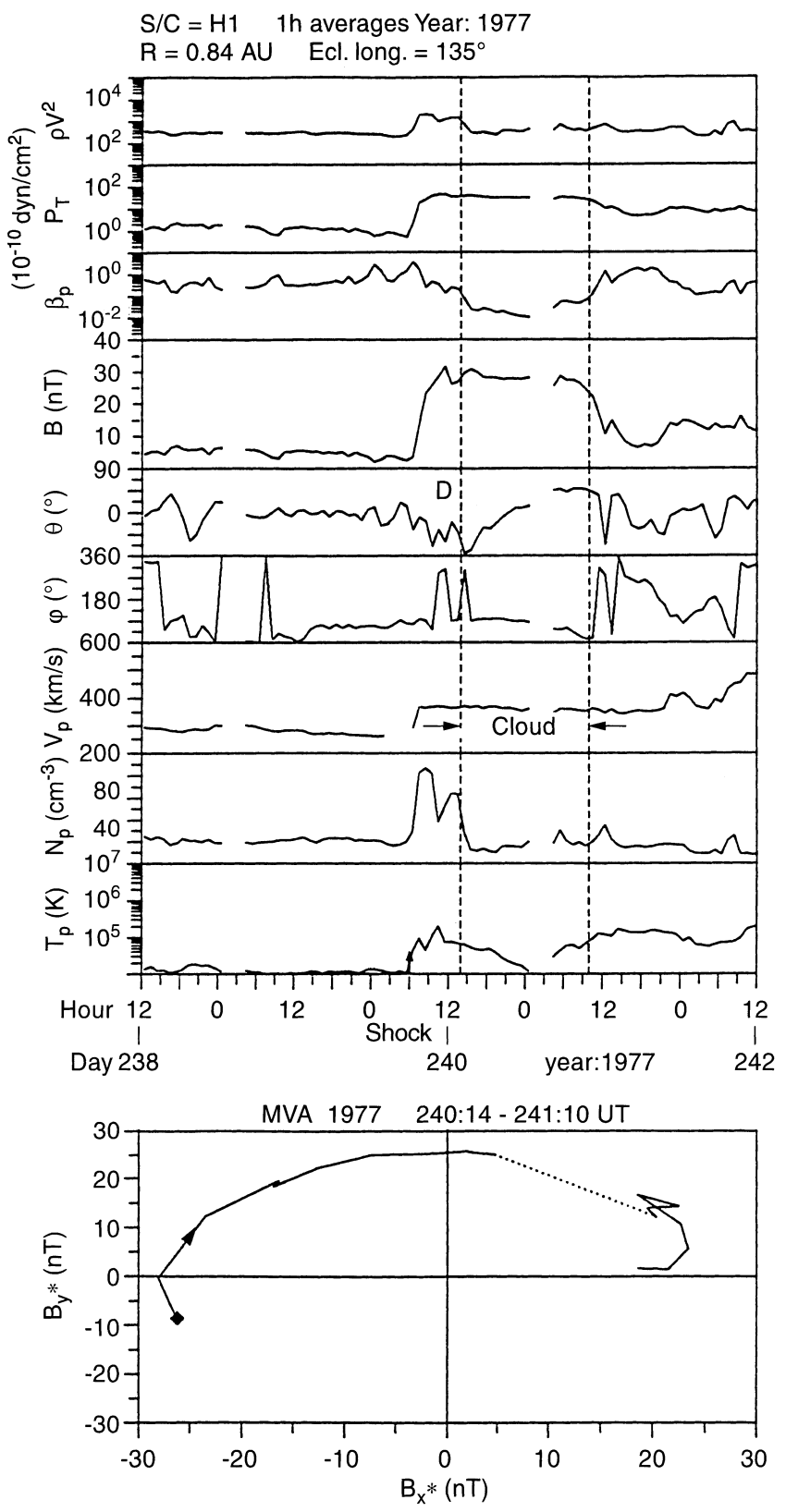

Fig. 5. A MC (dashed lines) of type SEN observed with Helios 1 at 0.8 AU on days 240,14 UT - 241, 10 UT in 1977. The MC was associated with a shock on day 240,06 UT. $D$ denotes the draping of the IMF in the cloud's sheath region. The bottom panel shows the clockwise rotation with $+B_{\mathrm{y}}$-values in the plane of maximum variance. The calculated axis orientation was $\varphi=112^{\circ}, \theta=+12^{\circ}$, with a minimum variance direction of $\varphi=205^{\circ}, \theta=+17^{\circ}$

inclined towards the ecliptic $\left(\theta=-17^{\circ}\right)$. The angles for the direction of minimum variance were $\varphi=$ $168^{\circ}, \theta=+26^{\circ}$.

\subsection{Statistical results}

For all $46 \mathrm{MCs}$ we determined their orientation and magnetic configuration (see Table 1). The frequency distribution of the calculated axis orientations in terms
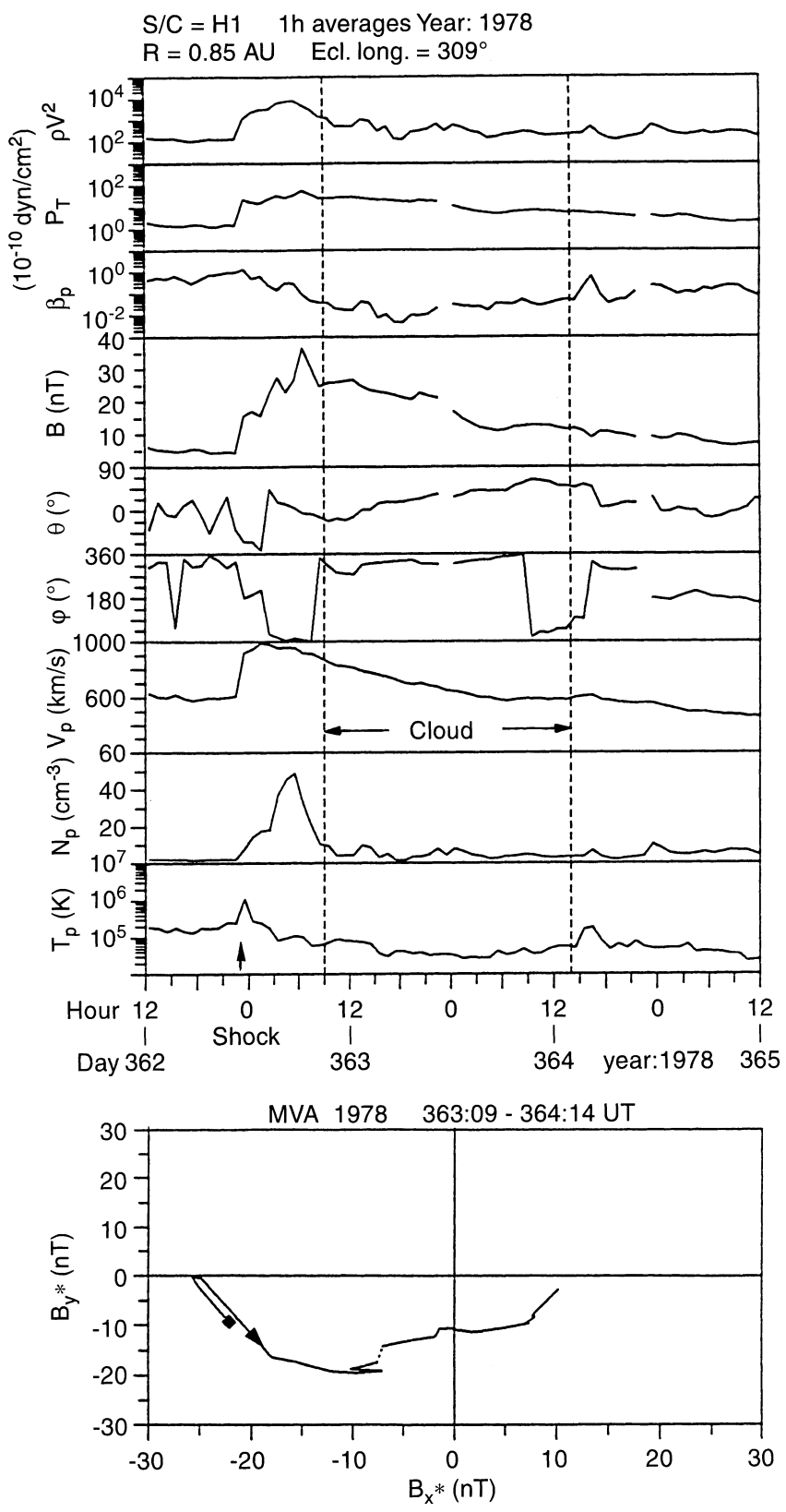

Fig. 6. A MC (dashed lines) of type SWN observed with Helios 1 at 0.9 AU on days 363, 09 UT-364, 14 UT in 1978, associated with a shock on day 362,23 UT. The bottom panel shows the counter-clockwise rotation with $-B_{\mathrm{y}}$-values in the plane of maximum variance. The cloud's axis direction was $\varphi=117^{\circ}, \theta=-30^{\circ}$, the minimum variance direction was $\varphi=230^{\circ}, \theta=+47^{\circ}$

of $\theta$ and $\varphi$ is shown in Fig. 9. In the top diagram of Fig. 9 we compared the frequency distribution of the axes inclinations to the ecliptic between $90^{\circ} \mathrm{S}-90^{\circ} \mathrm{N}$ found for the Helios 1/2 MCs with a random distribution of orientations for the same number of events in bins of $20^{\circ}$. Solid bars represent the number of MCs in each bin, open bars represent the number of cases as expected from the random distribution. Assume that the sky is divided into bins of equal areas of one square each, then for $\theta=0^{\circ}$ there are 360 possibilities for $\varphi$, but there is only one possibility for $\theta=90^{\circ}$. Thus random orientations at $0^{\circ}$ should be 360 times more likely than those at 

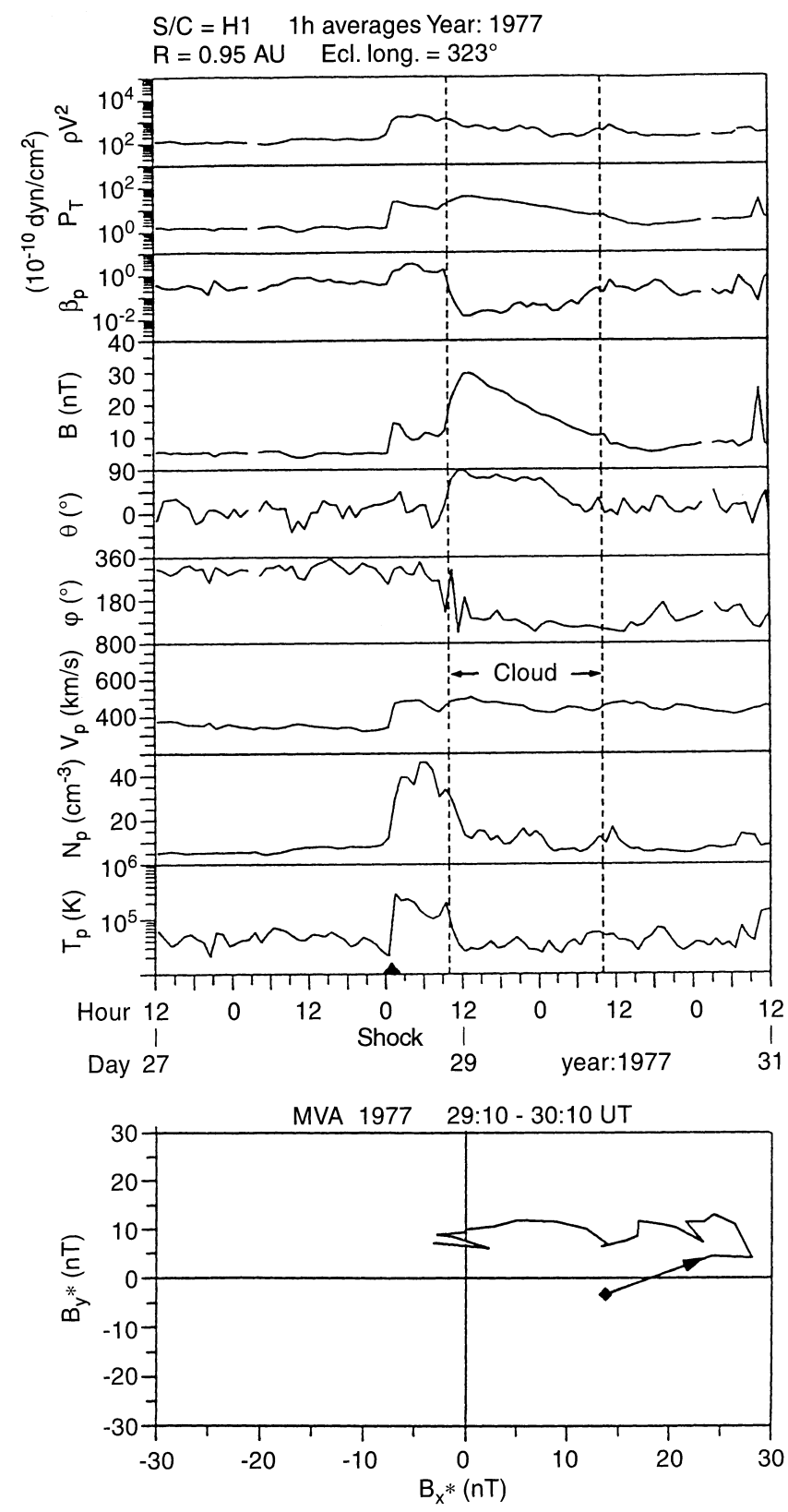

Fig. 7. A MC (dashed lines) of type NES observed with Helios 1 on days 29,10 UT - 30, 10 UT in 1977. It was preceded by a shock on day 29, 01 UT. The bottom panel shows the counter-clockwise "rotation" with $+B_{\mathrm{y}}$-values in the plane of maximum variance. The cloud's axis was $\varphi=103^{\circ}, \theta=+8^{\circ}$, the minimum variance direction was $\varphi=191^{\circ}, \theta=-15^{\circ}$

$90^{\circ}$. A more sophisticated approach shows that the area is proportional to $\int \vartheta$ for the measured range of $\vartheta$, where $\vartheta$ is the colatitude. Figure 9 shows that the distributions are roughly comparable, i.e., MCs can have in principal any inclination with respect to the ecliptic. However, there is an indication for an overabundance of axes inclinations in the range $10^{\circ} \mathrm{S}-10^{\circ} \mathrm{N}$. Since $\mathrm{MC}$ axis do not spread randomly over $180^{\circ}$ in azimuth the number of cases in the interval $10^{\circ} \mathrm{S}-10^{\circ} \mathrm{N}$ should be too peaked in the random distribution in Fig. 9 supporting the assumption that the axes of MCs commonly lie rather close to the ecliptic than highly inclined to it.
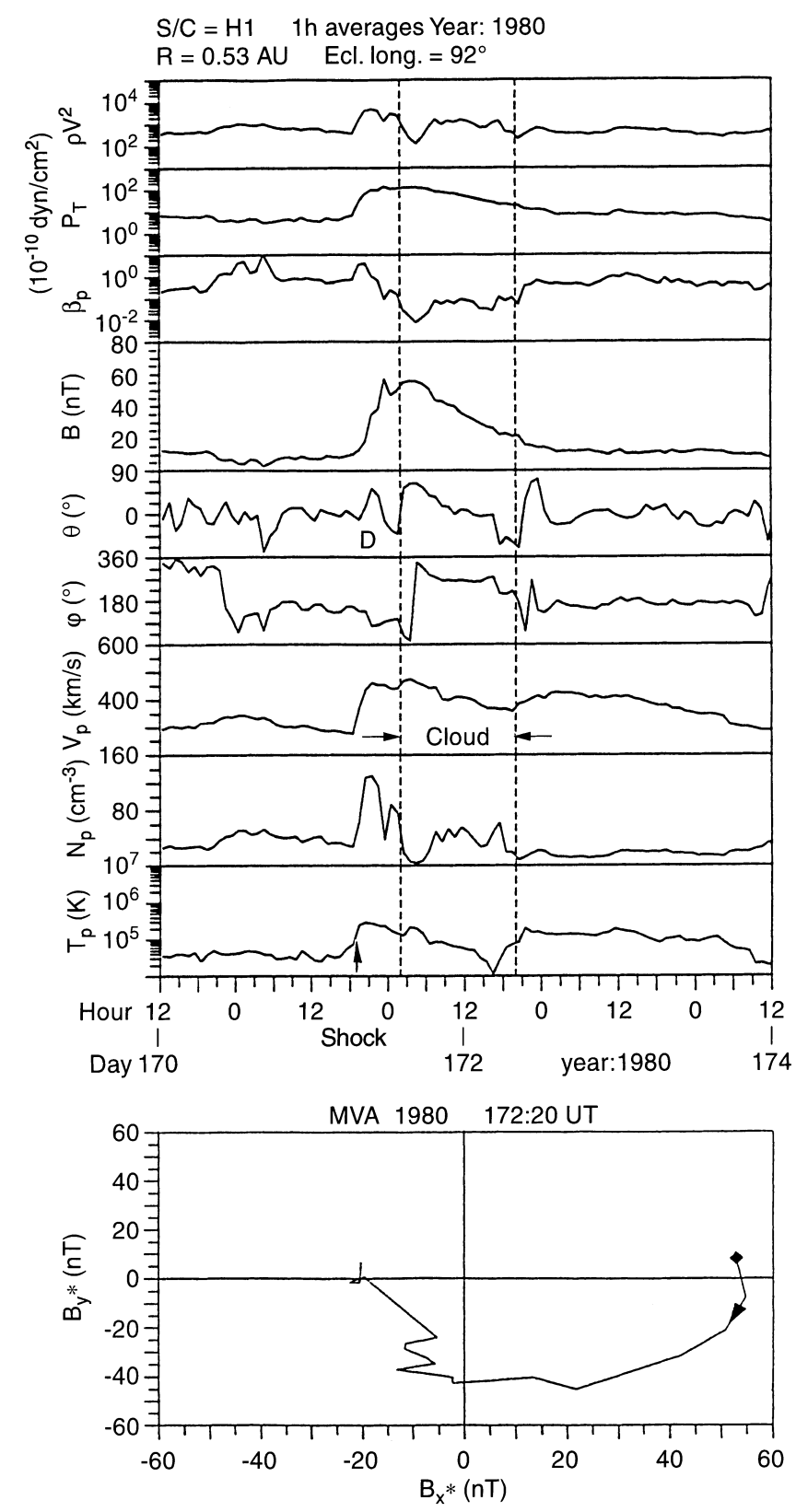

Fig. 8. A MC (dashed lines) of type NWS that passed Helios 1 at 0.5 AU on day 172, $02-20$ UT in 1980. It was associated with a shock on day 171,19 UT. $D$ denotes the draping of the IMF in the cloud's sheath region. The bottom panel shows the clockwise rotation with $-B_{\mathrm{y}}$-values in the plane of maximum variance. The calculated axis direction was $\varphi=87^{\circ}, \theta=-17^{\circ}$, the minimum variance direction was $\varphi=168^{\circ}, \theta=+26^{\circ}$

The distribution for the azimuthal axes direction of MCs in the ecliptic between $0^{\circ}-180^{\circ}$ is shown in the lower diagram of Fig. 9, also in bins of $20^{\circ}$. The axes scattered about the east-west direction, i.e., normal to the sun-spacecraft line. The average azimuthal orientation of the flux tube axes was $\langle\varphi\rangle=91^{\circ}$, rms $=44^{\circ}$, and the average orientation of the minimum variance direction was $\langle\varphi\rangle=188^{\circ}, \quad \mathrm{rms}=38^{\circ}$ and $\langle\theta\rangle=+3^{\circ}$, $\mathrm{rms}=27^{\circ}$. Our findings confirm the results for IPCs and shocks found by Smith and Phillips (1997). 

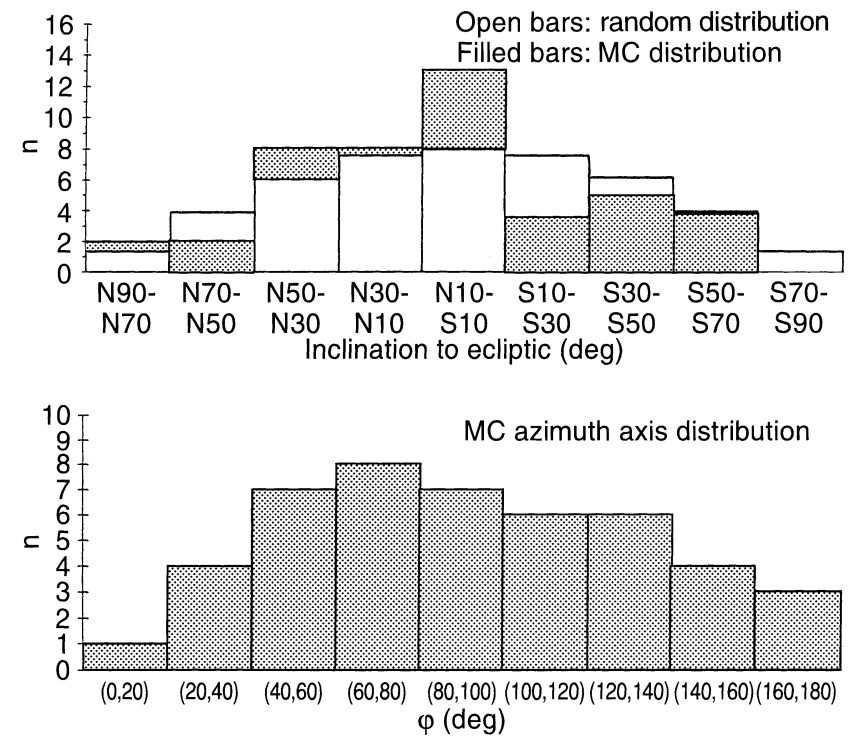

Fig. 9. Frequency distribution for the calculated axis orientations of all $46 \mathrm{MCs}$ observed with Helios $1 / 2$ between 0.3 and 1 AU. Top diagram: comparison of the distribution for the cloud's axis inclinations relative to the ecliptic plane in $20^{\circ}$ bins between $90^{\circ} \mathrm{S}-90^{\circ} \mathrm{N}$ with a random distribution. Solid bars represent the number of MCs in each bin, open bars represent the number of cases expected from the random distribution. Bottom diagram: distribution for the cloud's axis azimuthal angles in the ecliptic in $20^{\circ}$ bins between $0^{\circ}-180^{\circ}$

Most of the clouds' axes were lying inside a cone angle of $\pm 45^{\circ}$ with respect to the east-west direction in the ecliptic, distinctive from the average Parker spiral direction of the $\operatorname{IMF}\left(\varphi \approx 135^{\circ}\right.$ at 1 AU $)$. The large scatter of the axes orientations around the east-west direction may result from passage through the individual MCs under different aspect angle relative to the center of the MCs. Often left or right wings of the flux tubes may have been encountered by the Helios probes. BDEs seem to be best explained through heat fluxes of supra-thermal electrons along magnetic field lines that remain connected back at both ends to the solar corona (e.g., Gosling, 1990) so that expanding flux tubes should in reality be curved obstacles, which could explain the observed scatter of the axes orientations in the azimuthal direction.

Table 2 shows the frequencies of the different flux rope types observed by Helios $1 / 2$. SEN- and SWNclouds were observed approximately three times more frequently than NES- and NWS-type MCs during 1974 to 1981 . On the other hand, comparing the frequency of MCs by magnetic helicity, the number of left-handed (SEN, NWS) and right-handed (SWN, NES) MCs was the same, as one might expect if these types occur with equal probability. Zhang and Burlaga (1988) found that $79 \%$ of the MCs they investigated for the years 19781982 were associated with SN-rotations of the magnetic field vector, but they did not gave a physical explanation for this preference.

A time-period of the solar cycle similar to the years 1974-1981 is 1984-1991. For these years we inspected the near Earth solar wind data (OMNI-database at
Table 3. Frequencies of SN- and NS-MCs at Earth's orbit during 1984-1991. The dates and types of the individual MCs are given below the Table

\begin{tabular}{lll}
\hline Years & SN-MCs & NS-MCs \\
\hline $1984-1991$ & 2 & 7 \\
\hline
\end{tabular}

(dates and types: 1 May 85, NS; 12 Jul 85, SN; 24 Nov 86, NS; 14 Jan 88, NS; 21 Feb 88, NS; 14 Jun 89, NS; 29 Aug 89, NS; 25 Apr 91, NS; 9 Jul 91, SN)

NSSDC, for a description of this data set see King, (1991), for MCs showing a unique coherent internal SNor NS-rotation of the magnetic field vector according to the identification criteria of MCs at 1 AU (Burlaga, 1991). Our search yielded only nine clearly defined MCs, since the data are sparse and we did not investigate events with complex structure or time durations shorter than $\sim 1$ day. Table 3 shows that seven of the nine MCs identified between 1984-1991 had NS-rotations, contrary to the years 1974-1981 where SN-MCs were dominant.

For the years 1974-1981 (solar cycle 21), magnetic field lines in the Sun's northern hemisphere had predominantly northern magnetic polarity, i.e. they were pointing away from the Sun, whereas magnetic field lines in the Sun's southern hemisphere were predominantly directed sunwards (southern magnetic polarity). During 1984-1991 (cycle 22) the magnetic polarities in the Sun's hemispheres were reversed. The observations are in agreement with the findings of Bothmer and Rust (1997) who suggested a solar cycle dependence of the magnetic configuration of MCs. This topic will be further discussed in Sec. 8, 9.

\section{Large-scale structure of MCs}

MCs are thought to be large-scale solar wind structures. Thus, they should be observable over a substantial range in solar longitude/latitude with the same overall magnetic flux tube structure. One spectacular event in which the same MC was observed by Helios, Voyager and near-Earth spacecraft at distances between 1-2 AU has been studied in detail by Burlaga et al. (1990). The authors concluded that the spacecraft observations are in agreement with the model of a MC as a large-scale magnetic flux-rope.

To further clarify the structure of MCs, we investigated near-Earth solar wind data collected in the OMNI-database at NSSDC to see if the MCs observed by Helios 1/2 also passed the Earth (see Table 1). In a few cases, when only plasma data were available and when the MC observed by the Helios s/c had a substantial southward magnetic field, we additionally investigated geomagnetic activity which sensitively responds to large southward components of the IMF (see, e.g., Bothmer and Schwenn, 1995). Doubtful events due to major gaps in the solar wind data and other uncertainties were discarded, leaving 9 cases (note in Table 1 that Helios 1 and Helios 2 observed the same 

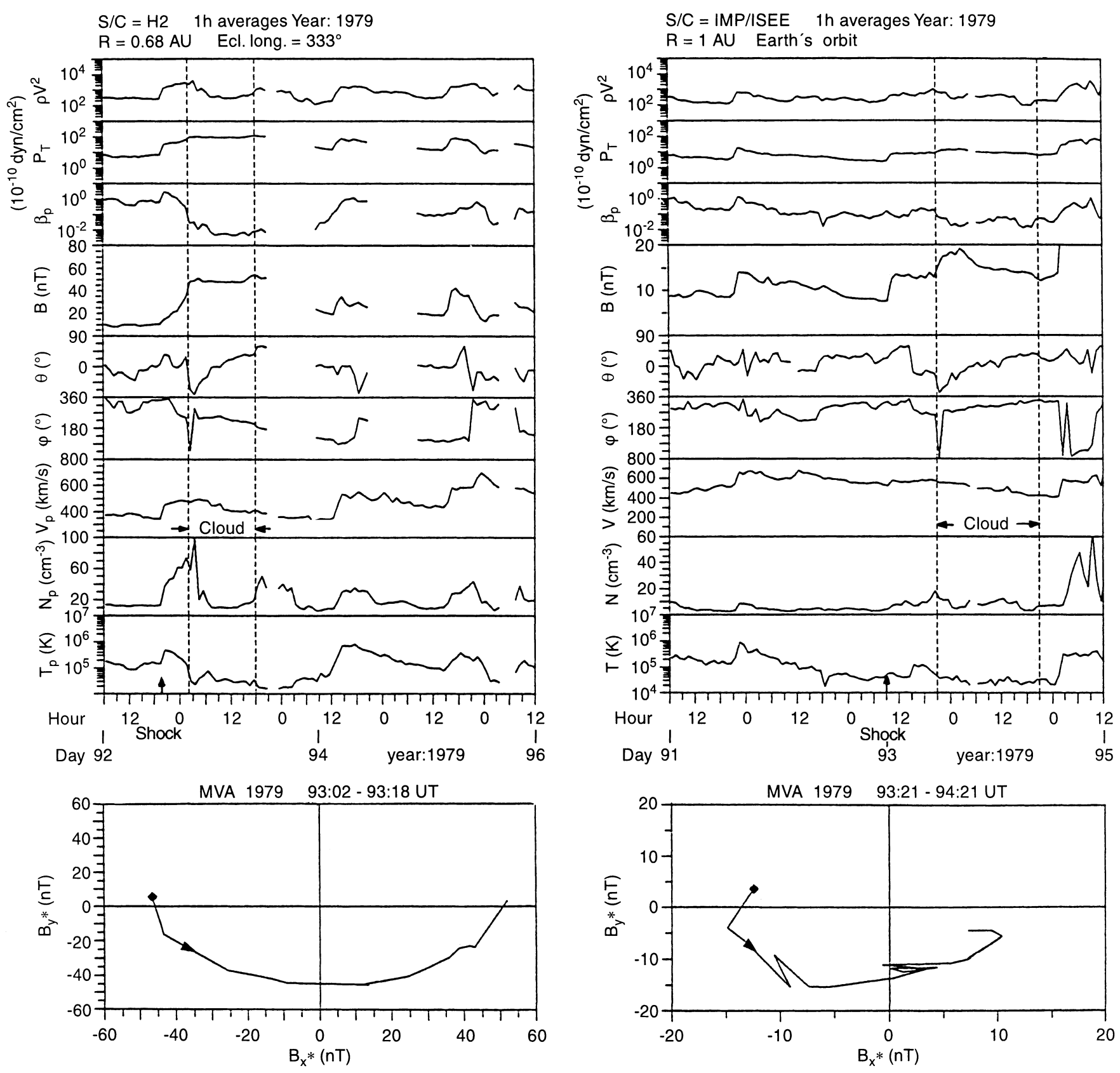

Fig. 10. Solar wind parameters of a MC (dashed lines) that passed Helios 2 at $0.7 \mathrm{AU}, 27^{\circ} \mathrm{E}$ of Earth on day 93, 02-18 UT (left panel) and Earth's orbit on days 93, 21 UT - 94, 21 UT (right panel). Times

MC in the events in January and February 1978). Figure 10 gives an example. The left panel shows a MC that was observed by Helios 2 in April 1979 at 0.7 AU, $27^{\circ}$ east of the Earth. Due to data gaps it was difficult to define the rear boundary of the MC unambiguously. The time interval that we identified as the MC is comparable to the start- and end-times given for this event by Burlaga et al. (1987).

This MC later passed Earth's orbit as shown by IMP/ ISEE observations in the right panel. The MC was associated with an interplanetary shock and caused a strong geomagnetic storm with $\mathrm{Kp} \geq 8$ - (see also Burlaga et al., 1987). From the results of the MVA the cloud

of the associated shocks: day 92, 20 UT at Helios 2; day 93, 09 UT at IMP/ISEE. Results from the MVA of the magnetic field data are displayed in the bottom panels

was identified as a flux tube of type SWN. The spacecraft measurements of IMP/ISEE and that of Helios 2 show a striking similarity, although the MC was observed in different ambient solar wind streams. At Earth's orbit the MC was propagating through fast ambient wind, whereas it plowed through low speed wind at Helios 2. This observation is consistent with the slightly higher plasma speed of the MC at Earth's orbit $(\mathrm{V} \sim 500 \mathrm{~km} / \mathrm{sec})$, compared with the plasma speed measured at Helios $2(\mathrm{~V} \sim 450 \mathrm{~km} / \mathrm{sec})$. Note that Helios 2 observed the MC at a sector boundary (see the change in $\varphi$ from $\sim 315^{\circ}$ to $\sim 135^{\circ}$ in Fig. 10). The orientation of the cloud's axis as calculated from the minimum 
variance method was $\varphi=79^{\circ}, \theta=+14^{\circ}$ (time interval: 93:02-93:18 UT) at Helios 2 and $\varphi=88^{\circ}, \theta=-12^{\circ}$ (time interval: 93:21-94:21 UT) at Earth's orbit with minimum variance directions of $\varphi=164^{\circ}, \theta=-19^{\circ}$ at Helios 2 and $\varphi=173^{\circ}, \theta=+22^{\circ}$ at Earth's orbit.

Figure 11 is a simple sketch for the possible largescale configuration of this $\mathrm{MC}$ at the arrival time of the $\mathrm{MC}$ at Helios 2 based on the calculated axis orientations at the positions of Helios 2 and at Earth's orbit. The axis orientation of the MC at the heliolongitude of the Earth at the arrival time of the MC's center at Helios 2 was determined back by using the speed of the $\mathrm{MC}$ measured at $1 \mathrm{AU}$ and the time delay between the MC's arrival at Helios 2 and 1 AU, assuming that the MC propagated along the Sun-Earth line with constant speed. Note that Helios 1 at $0.7 \mathrm{AU}, 43^{\circ}$ east of Helios 2, did observe the shock, but no signatures of a MC or ejecta in general could be found in the data.

In nine cases where a MC was detected from both Helios and Earth-bound satellites, longitudinal separations of up to $\sim 60^{\circ}$ occurred. We find that in cases with separations $>60^{\circ}$, the $\mathrm{MC}$ was observed by only one spacecraft. Figure 12 shows the histogram obtained from our study. In two cases, in which both Helios s/c observed the same MC, we used the value of the spacecraft which was separated largest in longitude from Earth. Based on these observations the azimuthal broadness of a $\mathrm{MC}\left(60^{\circ}\right.$ in heliolongitude $\simeq 1 \mathrm{AU}$ at 1 AU) should be larger than its radial size (the typical diameter of a $\mathrm{MC}$ is $\sim 0.25 \mathrm{AU}$ at $1 \mathrm{AU})$. Figure 12 might imply a smaller extent for MCs in heliolongitude compared to the value of $\sim 100^{\circ}$ found previously for shock-associated IPCs (Richardson and Cane, 1993), but we have not found any case where a MC was observed with two spacecraft separated by more than $60^{\circ}$. A possible explanation for this discrepancy could be that the events studied by Richardson and Cane (1993)

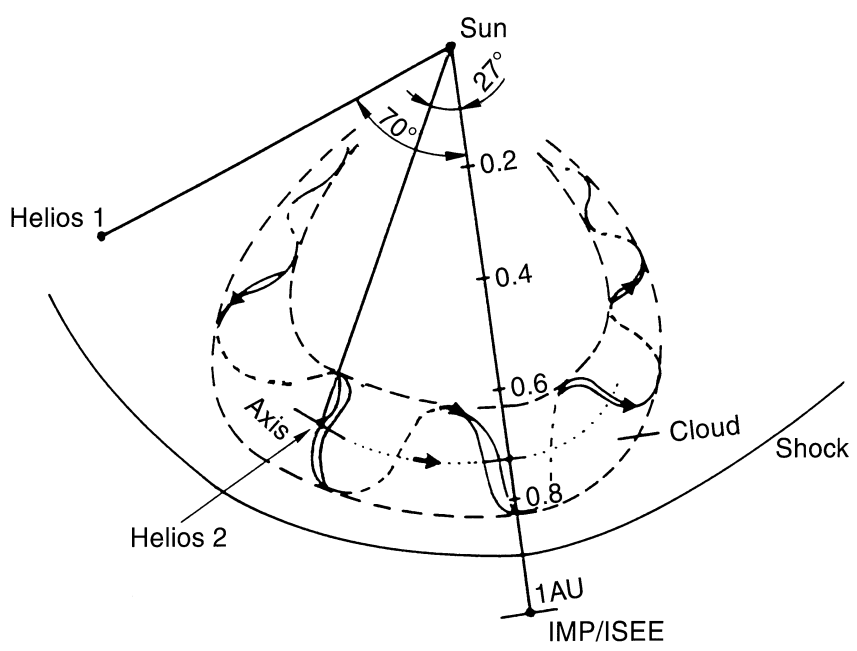

Fig. 11. Sketch showing the possible large-scale geometry of the MC observed by Helios 2 and IMP/ISEE in April 1979 (see Fig. 10) based on results of the MVA of the magnetic field data. Helios 1 did observe the shock, but not the MC. Arrows denote the orientation of the magnetic field lines at the cloud's outer boundaries and on its axis

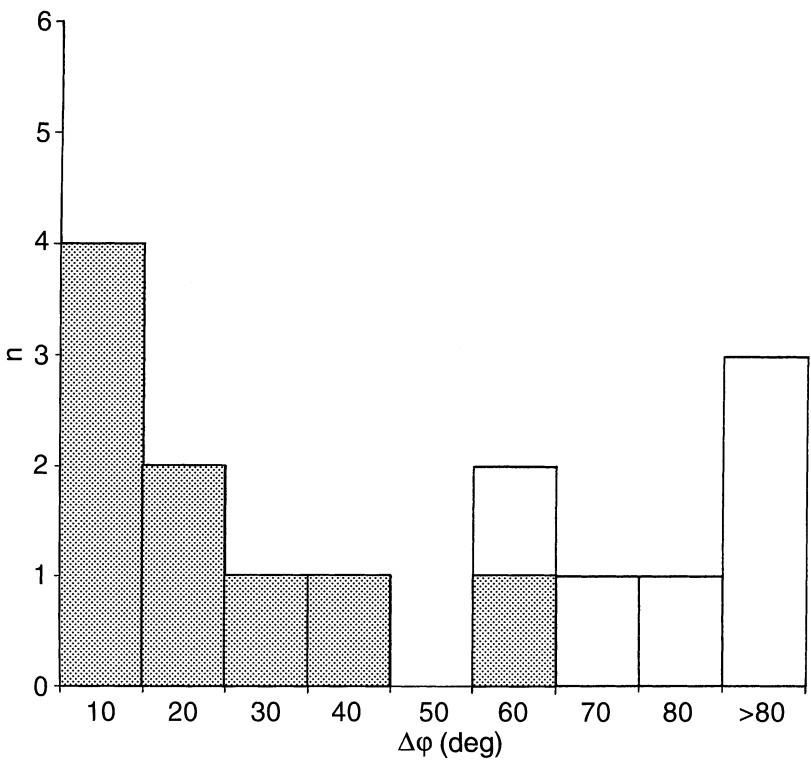

Fig. 12. Frequency distribution for the longitudinal span of MCs in the ecliptic based on correlated Helios and near Earth spacecraft observations. The spacecraft's longitudinal separation, $\Delta \varphi$, is given in intervals of $10^{\circ}$. Observations for longitude separations of more than $80^{\circ}$ have been added together. Solid bars mark the number of events in which a MC passed both spacecraft separated by the given longitude interval in the ecliptic. Empty portions of the bars denote cases in which MCs were not observed by the s/c at Earth's orbit at the given angular separations from the Helios $\mathrm{s} / \mathrm{c}$

were all flare-associated and thus probably more energetic and larger in their spatial extent.

\section{Expansion of Magnetic clouds}

Burlaga and Behannon (1982) investigated MCs in the outer heliosphere using Voyager data. They found larger radial sizes for MCs observed further away from the Sun. To investigate the expansion of MCs in the heliosphere, we used the MCs observed by Helios $1 / 2$ between 0.3-1 AU and investigated whether they had passed also the Voyager spacecraft in the outer heliosphere (s/c data other than Helios were taken from the COHO data base at NSSDC, described, e.g., in NSSDC News vol. 8(2), 1992). We found a number of cases in which the same MC could be uniquely identified in both data sets with time delays corresponding to the outward propagation of the MCs from the Helios to the Voyager spacecraft with approximately constant speed.

Here we present an example observed by Helios 1 at 0.9 AU and by Voyager 1/2 at 2.6 AU in March 1978 (for the identification of the MC in the Voyager data see also Burlaga and Behannon, 1982). The left side of Fig. 13 shows magnetic field data for Helios 1 (top panel) and Voyager 1/2 (middle, bottom panel), and the right side shows the corresponding results of the MVA. The spacecraft were separated by $18^{\circ}$ in solar longitude and by 1.6 AU in solar distance. No signatures of a solar wind transient were found in the plasma data at Earth's orbit, $55^{\circ}$ west of Helios 1 , i.e., $73^{\circ}$ west of Voyager $1 / 2$, 

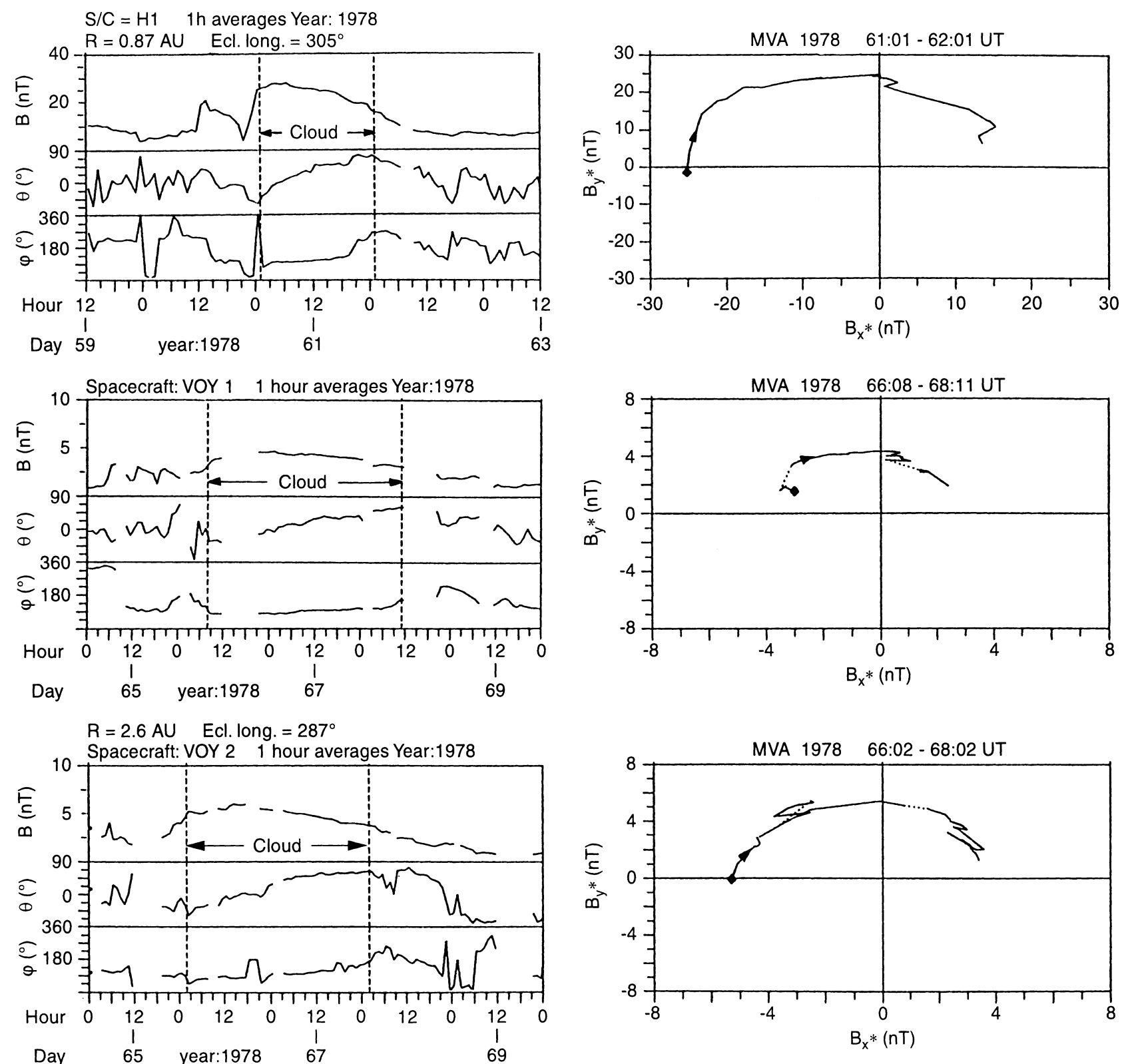

Fig. 13. Magnetic field data and results of the MVA for a MC (dashed lines) that passed Helios 1 at 0.9 AU on days 61, 01 UT - 62, $01 \mathrm{UT}$, Voyager 1 at $2.6 \mathrm{AU}$ on days $66,08 \mathrm{UT}-68,11 \mathrm{UT}$ and

Voyager 2 on days 66, 02 UT - 68, 02 UT in 1978. The Helios and Voyager spacecraft were separated by $18^{\circ}$ in solar longitude. Note the longer time duration of the MC at Voyager 1/2

so that we can assume that there was also no $\mathrm{MC}$ at the Earth although there was a gap in the magnetic field data. Note the striking similarity of the directional changes of the magnetic field vector at all three spacecraft, which all detected a SEN-type MC. The arrival time of the MC at Voyager 1, at 2.6 AU (08 UT on day 66) and at Voyager 2 (02 UT on day 66) agreed well with the propagation time from Helios 1 to Voyager $1 / 2$ as estimated from the plasma speed of $\sim 446 \mathrm{~km} / \mathrm{sec}$ observed during the cloud's passage over Helios 1 at 0.9 AU. The cloud showed a much longer time-duration at the position of Voyager $1(t=50 \mathrm{~h})$ and Voyager 2 $(t=48 \mathrm{~h})$ compared to Helios $1(t=24 \mathrm{~h})$, thus giving direct evidence for the expansion of this MC. The MVA yielded similar results for the orientation of the cloud's axis at all three spacecraft, with values of $\varphi=119^{\circ}$, $\theta=+44^{\circ}$ at Helios $1, \varphi=91^{\circ}, \theta=+30^{\circ}$ at Voyager 1 , and $\varphi=83^{\circ}, \theta=+28^{\circ}$ at Voyager 2 .

Figure 14 presents a log-log plot showing the growth of the radial sizes of MCs between 0.3 and 4.2 AU obtained from Helios 1/2, Voyager 1/2, and Pioneer 10 data (Bothmer and Schwenn, 1994). The smallest MCs had radial sizes $<0.1 \mathrm{AU}$ and were observed close to the Sun, the largest MCs had radial sizes $>0.4 \mathrm{AU}$ and were observed beyond 2 AU.

From linear regression it is found that the radial size, $\mathrm{s}(R)$ in $\mathrm{AU}$, of MCs increases with radial distance, $R$, from the Sun as: 


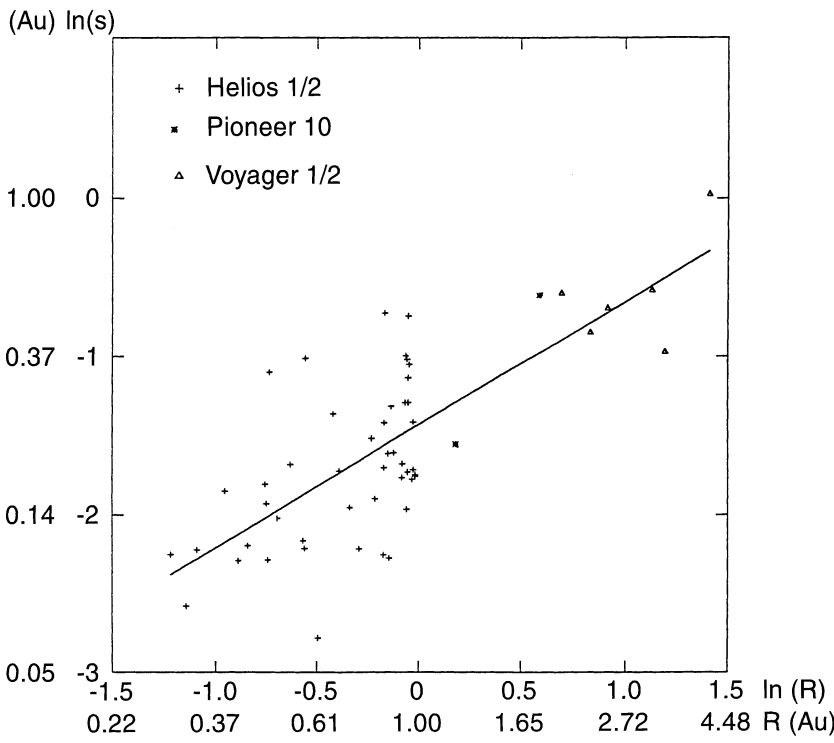

Fig. 14. Double logarithmic plot showing the radial sizes, $s$, of MCs observed by Helios $1 / 2(+)$, Voyager $1 / 2(\Delta)$, Pioneer $10(*)$ between 0.3 and 4.2 AU, versus solar distance, $R$ (from Bothmer and Schwenn, 1994). The straight line was obtained by linear regression: $s(R)=(0.24 \pm 0.01) \times R^{0.78 \pm 0.10}[\mathrm{AU}]$

$$
\mathrm{s}(R)=(0.24 \pm 0.01) \times R^{(0.78 \pm 0.10)}(R \text { in } \mathrm{AU})
$$

There is a clear trend for an increase in the radial sizes of MCs with distance from the Sun, confirming that radial expansion is a common feature of MCs in the heliosphere.

MCs observed considerably closer to the Sun compared to $1 \mathrm{AU}$ had often higher plasma densities than the surrounding solar wind (see Fig. 1). The density inside the flux tubes might fall off rapidly with increasing distance from the Sun as indicated by the radial expansion found for MCs. We determined the average proton density, $\left\langle N_{P}\right\rangle$, inside each of the MCs observed by the Helios probes. Figure 15 shows in a log-log plot $\ln \left(\left\langle N_{p}\right\rangle\right)$ versus $\ln (R)$. For comparison the decrease of the proton density measured in slow $(V<400 \mathrm{~km} / \mathrm{s}$; dashed line) and fast $(V>600 \mathrm{~km} / \mathrm{s}$; dotted line $)$ solar wind streams over the range $0.3-1$ AU has been included. These slopes were calculated according to results summarized in Schwenn (1990) with $\left\langle N_{p}\right\rangle=N_{p}$ $\times R^{-2.1} \mathrm{~cm}^{-3}$ ( $R$ in $\left.\mathrm{AU}\right)$.

From linear regression we found that the density of MCs decreases with increasing radial distance, $R$, as:

$N_{p}=(6.47 \pm 0.85) \times R^{(-2.4 \pm 0.3)} \mathrm{cm}^{-3}(R$ in $\mathrm{AU})$

Thus the density inside MCs decreases stronger with radial distance from the Sun than that of the average solar wind which falls off as $R^{-2.1}$ in agreement with what one would expect for expanding structures. Figure 15 shows that the density in MCs is generally higher than that of the average fast solar wind and that it is also generally higher than that of the slow solar wind at closer distances to the Sun. Note that this result implies, without taking density enhancements into account that have been caused by compression due to

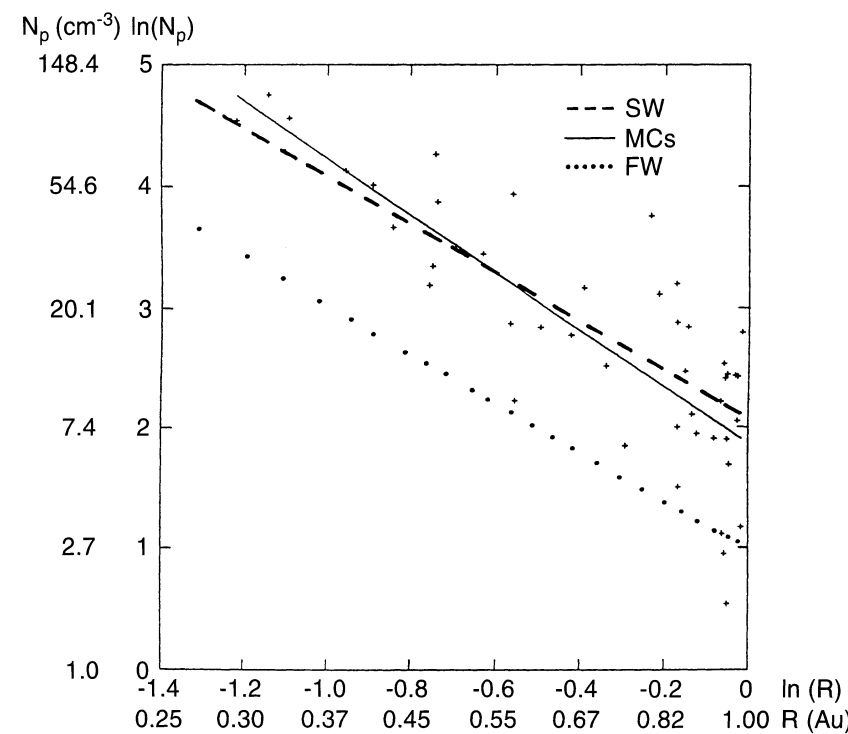

Fig. 15. Double logarithmic plot showing the average proton density inside MCs observed by Helios $1 / 2$ between 0.3 and 1 AU during 1974-1981. The straight line was obtained by linear regression: $N_{p}(R)=(6.47 \pm 0.85) \times R^{-2.4 \pm 0.3} \mathrm{~cm}^{-3}(R$ in AU). Dashed and dotted lines represent slopes for proton densities measured by the Helios probes in slow $(\mathrm{S})$ and fast $(\mathrm{F})$ solar wind-streams in the inner heliosphere (taken from Schwenn, 1991)

the interaction of a $\mathrm{MC}$ with the ambient solar wind, that IPC type MCs should be observed as transient white-light depletions rather than enhancements in the interplanetary medium at larger ( $\gtrsim 0.5 \mathrm{AU})$ distances from the Sun, in agreement with the suggested higher preference for MC associations with photometer IPCs in Table 1 for MCs observed closer to the Sun.

MCs in which the densities are found to be considerably lower compared to that of the ambient slow solar wind should have undergone strong expansion on their way out from the Sun. Consistent with this result, recent observations of the Ulysses spacecraft showed that IPCs including MCs were over-expanding at high latitudes in high speed solar wind due to their higher internal pressure compared to the surrounding flow (Gosling et al. 1994a,b).

To test whether our results are consistent with the flux-tube model for MCs, we assume that a MC has the topology of an ideal cylindrical flux tube of length 1 and diameter s, so that its volume $V$ is proportional to $\mathrm{s}^{2} \times 1$. We assume further mass is conserved inside the flux tube, i.e. $V \sim 1 / N_{p}$. From Eq. (1) the diameter of the flux tube increases due to the expansion as $R^{0.78}$. Based on observations of prominence eruptions and white-light observations from space-coronagraphs (see e.g. Webb and Hundhausen, 1987; Webb, 1988) we assume that 1 increases linearly with $R$. Thus, the volume of the flux tube should increase as $R^{2.56}$, i.e., the density should decrease as $R^{-2.56}$. If we consider the simplicity of the model assumptions, we find this value to be in qualitative agreement with the $R^{-2.4}$ dependence in Eq. (2). The present results obtained for MCs observed in the ecliptic by Helios $1 / 2$ are in agreement with the possible largescale topology of a MC sketched in Fig. 11. 


\section{Magnetic clouds and disappearing filaments}

Studies of the relationship of SMEs with other features of solar activity have shown that SMEs are better associated with prominence eruptions (disappearing filaments) than with solar flares (Harrison, 1986; Webb and Hundhausen, 1987). Yet, we do not know what causes SMEs nor which processes lead to the specific topology of the subset of SMEs associated with MCs in the interplanetary medium. A possible explanation is that a SME is caused by magnetic nonequilibrium leading to the rise of a prominence (e.g., Hundhausen et al. 1994; Priest, 1988; Feynman and Martin, 1995). The typical three-part structure of SMEs seen in white-light images (see, e.g., Webb, 1988 and references therein) is interpreted as a leading bright front comprised of coronal field lines with a cavity beneath it, followed by the rising prominence in the trailing portion of the SME.

Erupting quiescent prominences often show a magnetic flux-rope topology, so that it may seem plausible to speculate whether they are related to MCs (the term "eruption" means here that a prominence disappears in the $\mathrm{H} \alpha$-observations, i.e., an erupting prominence might remain attached to the Sun). The concept of force-free magnetic flux tubes was first introduced by Lüst and Schlüter (1954) for quiescent solar prominences and was later applied to MCs (Goldstein, 1983; see also the review on magnetic flux ropes given by Priest, 1990 and the paper on "theory of prominence eruption and propagation" by Chen, 1996).

The 'Catalog of Solar Filament Disappearances 1964-1980' (Wright, 1991) offered us the most useful data set to investigate whether quiescent filaments (disparition brusques, DBs) and MCs can be associated and to compare their magnetic structures (see also Bothmer, 1993; Rust, 1994). In order to establish unique associations between MCs that were observed in situ in interplanetary space by the Helios probes and DBs observed from Earth we used the following criteria: (1) Since the observations of DBs were taken from Earth we did not include any MC that was observed by the Helios probes when it was positioned further away than $60^{\circ}$ in solar longitude from the Sun's central meridian (as seen from Earth), in order to avoid DB-events which might have gone undetected due to limb darkening or because the DB might have occurred behind the Sun's limbs which reduced the number of events to 25 cases. (2) We regarded only those associations of MCs and DBs as safe when spacecraft and filament were separated not more than $\sim 20^{\circ}$ in solar longitude, and when the DBs had disappeared at the Sun in time windows (including the error in the given DB disappearance) that were calculated from the maximum and minimum proton speeds measured inside the MCs.

These criteria led to the $10 \mathrm{MC}$ events listed in Table 4. In five cases, MCs could be confidentially associated with a DB at the Sun and we compared their magnetic structures (see also Bothmer and Schwenn, 1994). The solar latitude of the filaments was $\sim 30-60^{\circ}$ (see Table 4). We remark that for the first two associations in Table 4 more than one filament disappeared during the estimated time windows for the solar events. In these cases the DB closest in longitude to the Helios probes has been selected. It is interesting to note that the daily rate of $\mathrm{DBs}$ between $45^{\circ} \mathrm{E}$ and $45^{\circ} \mathrm{W}$ was only $0.1-0.4$ during $1975-1980$.

Table 4. Associations between MCs observed with Helios $1 / 2$ and disappearing filaments (DBs). Left to right: date of the MC, s/c, s/c coordinates, minimum and maximum measured proton speeds inside the MC, calculated time-window for the solar event, disappearance of the DB at the Sun, solar position of the DB, magnetic polarity \& inferred flux tube type of the DB, in-situ observed type of the MC (after Bothmer and Schwenn, 1994)

\begin{tabular}{|c|c|c|c|c|c|c|c|c|}
\hline $\begin{array}{l}\text { MC } \\
\text { (year: } \\
\text { month: day) }\end{array}$ & $\mathrm{S} / \mathrm{C}$ & $\begin{array}{l}R[\mathrm{AU}] \\
\text { Long, Lat. } \\
\text { [deg] }\end{array}$ & $\begin{array}{l}V_{\min }, V_{\max } \\
{[\mathrm{km} / \mathrm{s}]}\end{array}$ & $\begin{array}{l}\text { Time-window } \\
\text { start-end } \\
\text { (month: day: } \\
\text { hour) }\end{array}$ & $\begin{array}{l}\text { DB } \\
\text { (month: day: } \\
\text { hour) }\end{array}$ & $\begin{array}{l}\text { Solar } \\
\text { position } \\
\text { [degree] }\end{array}$ & $\begin{array}{l}\text { Magnetic } \\
\text { polarity, } \\
\text { type }\end{array}$ & $\begin{array}{l}\text { MC } \\
\text { type }\end{array}$ \\
\hline 75:03:04 & $\mathrm{H} 1$ & $0.39 \mathrm{~W} 30, \mathrm{~S} 7$ & 418,507 & $\begin{array}{l}\text { 03:03:00 } \\
\text { 03:03:07 }\end{array}$ & no & & & \\
\hline $77: 12: 01$ & $\mathrm{H} 1$ & 0.75 E36, N5 & 401,431 & $\begin{array}{l}11: 28: 07 \\
11: 28: 12\end{array}$ & $11: 28: 06$ & $\begin{array}{l}\text { N60E65 } \\
\text { N50E33 }\end{array}$ & $-\backslash+$ SEN & SEN \\
\hline 78:01:17 & $\mathrm{H} 2$ & 0.98 E09, S4 & 305,340 & $\begin{array}{l}01: 11: 10 \\
01: 12: 00\end{array}$ & 01:11:09 & $\begin{array}{l}\text { S37W01 } \\
\text { S10W17 }\end{array}$ & $-/+$ NES & NES \\
\hline $78: 01: 29$ & $\mathrm{H} 2$ & 0.98 E13, S5 & 475,549 & $\begin{array}{l}01: 25: 21 \\
01: 26: 09\end{array}$ & 01:26:06 & $\begin{array}{l}\text { N55E25 } \\
\text { N39E08 }\end{array}$ & $-\backslash+$ SEN & SEN \\
\hline 78:02:06 & $\mathrm{H} 2$ & 0.98 E16, S5 & 367,444 & $\begin{array}{l}02: 02: 00 \\
02: 02: 19\end{array}$ & no & & & \\
\hline $78: 02: 16$ & $\mathrm{H} 2$ & 0.95 E19, S6 & 385,491 & $\begin{array}{l}02: 11: 19 \\
02: 12: 17\end{array}$ & $02: 11: 15$ & $\begin{array}{l}\text { S39E45 } \\
\text { S37E26 }\end{array}$ & $-/+$ NES & SWN \\
\hline 78:03:02 & $\mathrm{H} 1$ & $0.87 \mathrm{E} 55, \mathrm{~S} 4$ & 376,502 & $\begin{array}{l}02: 26: 00 \\
02: 27: 00\end{array}$ & no & & & \\
\hline 78:04:02 & $\mathrm{H} 2$ & $0.61 \mathrm{E} 17, \mathrm{~S} 7$ & 420,441 & $\begin{array}{l}03: 30: 12 \\
03: 30: 15\end{array}$ & 03:30:03 & $\begin{array}{l}\text { S60E39 } \\
\text { S45E09 }\end{array}$ & $+/-\mathrm{SWN}$ & SWN \\
\hline 79:04:03 & $\mathrm{H} 2$ & $0.68 \mathrm{E} 27, \mathrm{~S} 7$ & 389,496 & $\begin{array}{l}03: 31: 00 \\
03: 31: 16\end{array}$ & no & & & \\
\hline $79: 12: 31$ & $\mathrm{H} 2$ & $0.84 \mathrm{E} 15, \mathrm{~S} 1$ & 375,442 & $\begin{array}{l}12: 27: 02 \\
12: 27: 16\end{array}$ & no & & & \\
\hline
\end{tabular}



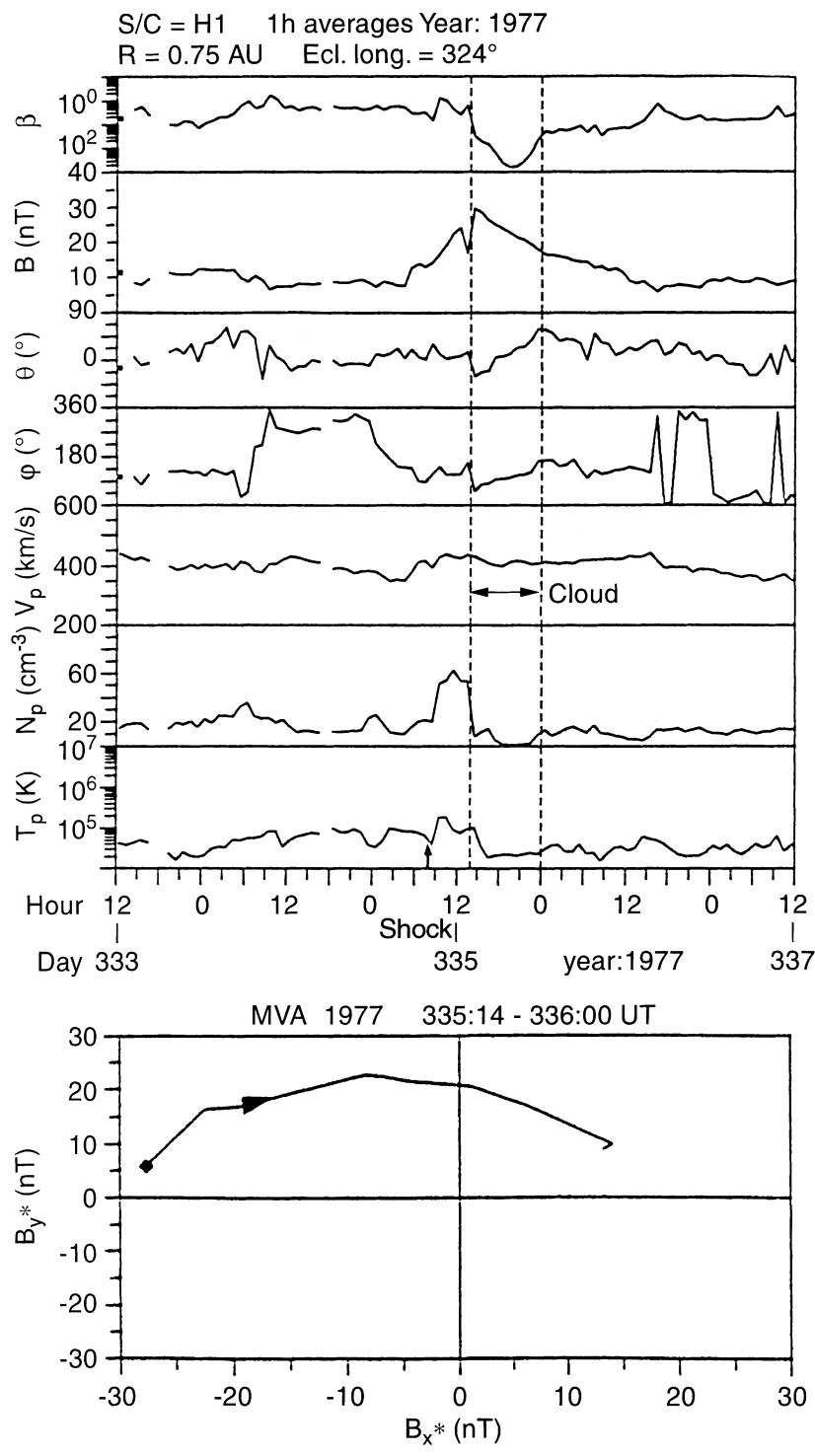

Figure 16 shows one of the five MCs of Table 4 that was associated with a DB. This MC was observed by Helios 1 in December 1977 at 0.8 AU. The figure shows the magnetic flux tube type as inferred from the MVA results and the inferred magnetic structure of the associated DB. The magnetic structure of the filament has been inferred from the polarity of the magnetic field with respect to the prominence axis based on the observation that the field structure in quiescent prominences is composed of loop-like arcades overlying the prominence axis (neutral line) (see, e.g., Dynamics and Structure of Quiescent Solar Prominences, edited by Priest, 1989), by taking into account the hemispheric magnetic patterns found for mid- to high-latitude filaments (Rust, 1967; Martin et al., 1994; Rust and Kumar, 1994a) and by applying the helicity rule found by Rust and Kumar (1994a) who inferred that for prominences in the northern solar hemisphere, the twist of field lines is preferentially left-handed, whereas it is preferentially right-handed for prominences in the southern hemisphere (compare with Fig. 18). This
DB: 28 November 1977, 06 UT

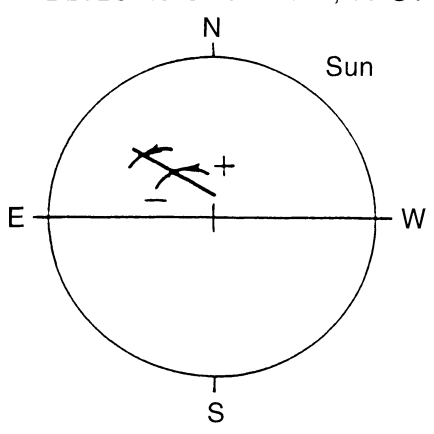

Fig. 16. An $\mathrm{MC}$ observed by Helios 1 at 0.8 AU (day 335, 14 UT - day 336, 0 UT) in December 1977 and the associated filament at the Sun. Upper left part: solar wind and plasma parameters for the MC. Upper right part: orientation and polarity of the filament $(D B)$ at the Sun (see Table 4). Left bottom panel: results from the MVA of the magnetic field data inside the MC. Right bottom panel: in situ observed flux tube type of the MC finding is consistent with sunspot observations which show that anti-clockwise whorls are three times as common in the north and clockwise ones in the south (Richardson, 1941; Yang et al., 1988). Anti-clockwise whorls are related to dextral and clockwise ones to sinistral filaments. It is interesting to note that the Helios observations showed SN-MCs to be three times more frequent than NS-MCs which is similar to the ratio of clockwise and anti-clockwise whorls in sunspots (Richardson, 1941).

The agreement of the magnetic topologies of the MC and the DB in Fig. 16 is striking. The individual fluxtube types given for the DBs in Table 4 have been labeled according to the notation that we introduced for MCs in Section 4 of this study (to compare with Table 2). Similar agreements between the magnetic structure of the filament and that of the associated MC were found in four of the five events listed in Table 4, with three different types of flux-tubes. In the one event in which we have not found an agreement, the MC had a complex internal magnetic field structure. 
Table 5. Associations between MCs observed with near Earth spacecraft (OMNI database) and DBs during 1978-1980. Left to right: date of $\mathrm{MC}$, speed range of $\mathrm{MC}$, date of associated $\mathrm{DB}$, solar position of $\mathrm{DB}$, magnetic polarity of DB, inferred DB flux tube type, MC flux tube type

\begin{tabular}{lllllll}
\hline MC (y:mo.:d) & $V(\mathrm{~km} / \mathrm{s})$ & DB (month:day) & Solar position & Magnetic polarity & Type & MC type \\
\hline 78:01:17 & $300-350$ & $01: 11$ & S37W01 S10W17 & $-/+$ & NES & NES \\
$78: 04: 03$ & $450-500$ & $03: 30$ & S60E39 S45E09 & $+/-$ & SWN & SWN \\
78:08:27 & $400-450$ & $08: 23$ & N16E18 N11E04 & +-- & NWS & NWS \\
$79: 04: 25$ & $500-600$ & $04: 22$ & S40E29 S36E07 & +- & SWN & SEN \\
$80: 02: 16$ & $350-450$ & $02: 11$ & N19E02 N17W22 & $-1+$ & SEN \\
$80: 03: 19$ & $300-400$ & $03: 16$ & S48W10 S53W32 & $-/+$ & NES \\
\hline
\end{tabular}

In the same way as for the Helios data, we have searched near Earth solar wind data from the OMNI and COHO data-bases at NSSDC for the years 19781980 when the data coverage was very good. We identified 12 MCs during these years. In four events DB observations were missing, one MC was not associated with a DB and in one event the filament was separated substantially in longitude from central meridian. Table 5 lists the six events that were found to be associated with a DB using the same criteria as for the Helios observations. Note that the first two events in Table 5 are MCs that have also been observed with the Helios probes so that Table 5 yields a total set of four new associations between MCs and DBs. We found an agreement between the magnetic flux tube type of the MC and that of the associated DB in all cases. Similar to the Helios observations, the filaments associated with the MCs, were observed typically at higher latitudes, in agreement with the recent findings by Bothmer and Rust (1997) based on an extended study of MCs observed at 1 AU and DBs.

Our results are summarized in Fig. 17 (adapted from Bothmer and Schwenn, 1994): four different magnetic flux tubes are expected in interplanetary space from the magnetic structure of disappearing filaments in the Sun's southern and northern hemispheres. The results indicate that the magnetic helicity of MCs observed in interplanetary space is the same as that of the associated filaments, i.e., the magnetic helicity inherent in magnetic fields at the Sun might be conserved outward into interplanetary space, as has been pointed out by Rust and Kumar (1994b) in agreement with findings of helicity conservation in laboratory plasmas. Note that on the contrary, Martin and McAllister (1997) claimed that the helicity of the overlying arches above a prominence axis which may evolve into flux tube is opposite to that of the filament beneath it.

Magnetic flux ropes/tubes may be formed through magnetic reconnection of coronal field lines in a rising SME as has been pointed out by Gosling (1990) and Gosling et al. (1995c,d). In support of this view, the Ulysses spacecraft has directly observed MCs and IPCs after formation of new coronal loops in solar regions that map to the spacecraft, as observed by the Japanese Yohkoh-satellite (Gosling et al., 1995c; Bothmer et al., 1996b; Lemen et al., 1996; Weiss et al., 1996). We believe that our findings are further evidence for an evolution of arcades of coronal loops into magnetic flux tubes (MCs). Weiss et al. (1996) found no characteristic

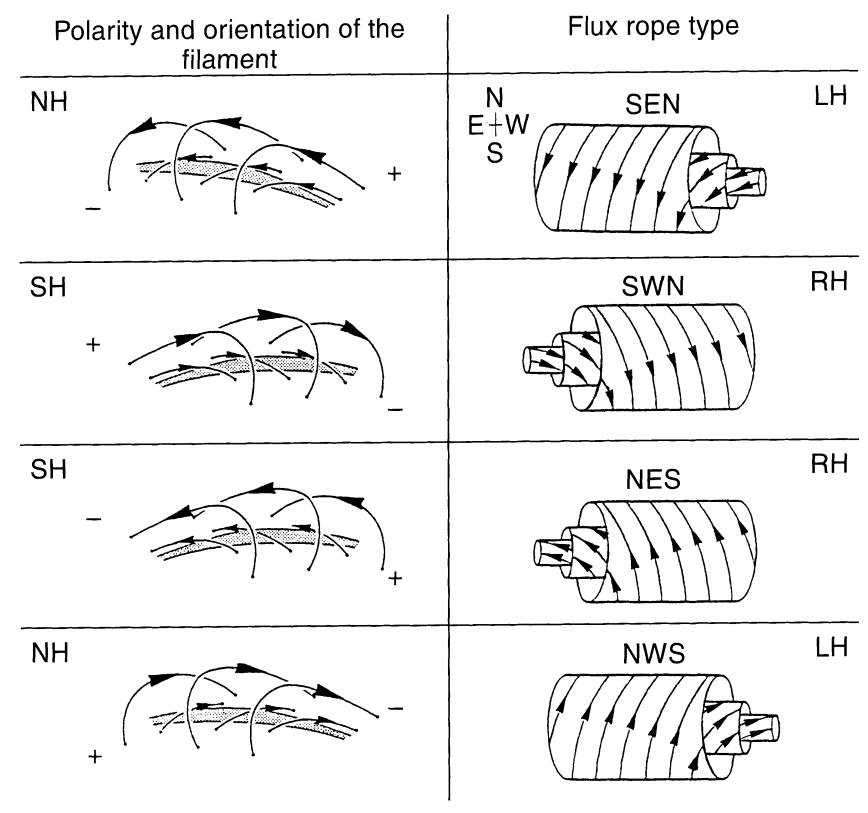

Fig. 17. Inferred magnetic structure of filaments in the Sun's northern and southern hemispheres $(\mathrm{NH}, \mathrm{SH})$ and that of associated MCs in interplanetary space (adapted from Bothmer and Schwenn, 1994). The abbreviations for the magnetic field structures have been chosen in analogy to the different flux tube types of MCs presented in Table 2. $L H$ (left-handed) and $R H$ (right-handed) denote the corresponding magnetic helicity

differences in the coronal soft X-ray signatures of IPCand MC-type IPC events, but they pointed out that the interplanetary plasma- $\beta$ of SMEs appears to be crucial in determining the magnetic field evolution. This is consistent with our finding of low plasma- $\beta$ values in MCs between $0.3-1$ AU. It is further interesting to remark that the magnetic fields of quiescent filaments are presumably low plasma- $\beta$ regions in the solar atmosphere (see, e.g., Priest, 1989).

We found evidence for a solar cycle variation of the magnetic structure of $\mathrm{MCs}$ in Sec. 5.2. In Fig. 18 (Bothmer and Rust, 1997) we propose an extended association between the polarity of sunspots, the magnetic structure of filaments and that of MCs, that could explain our observations.

It is well known that $\mathrm{H} \alpha$ filaments commonly occur in regions where sunspots are no longer visible. Usually the preceding sunspot (in the sense of solar rotation) lies closer to the solar equator than the following one which is of opposite magnetic polarity. The left column of 

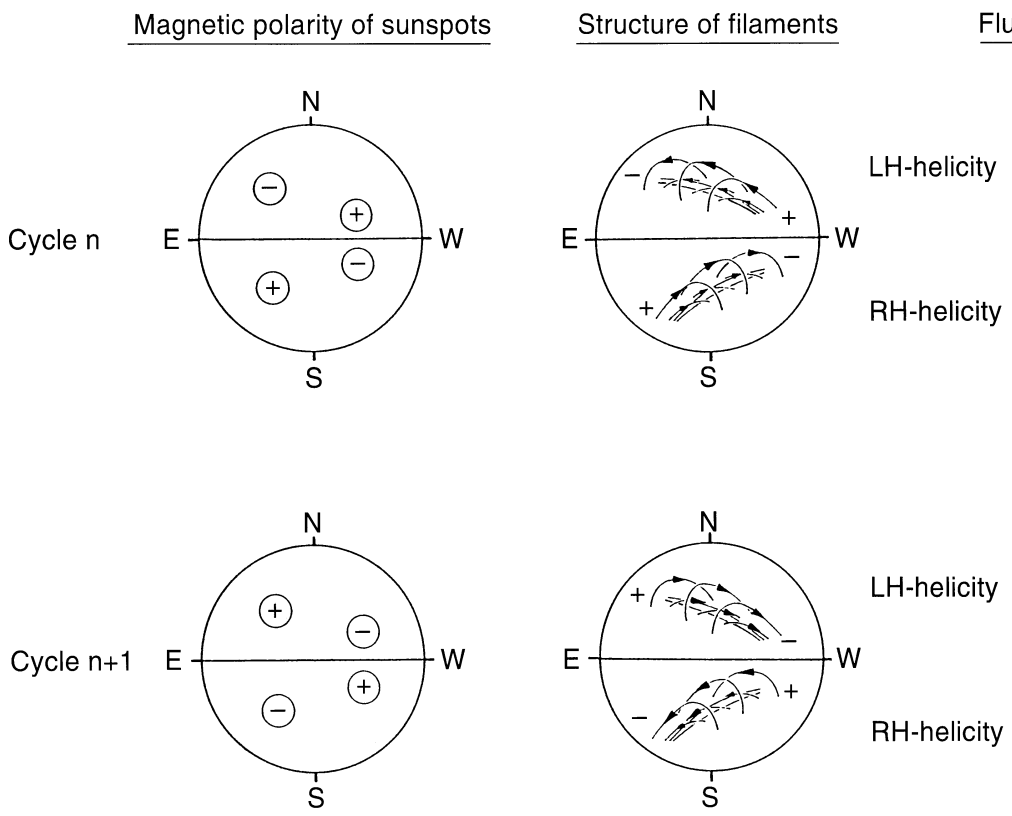

Flux rope type of magnetic clouds

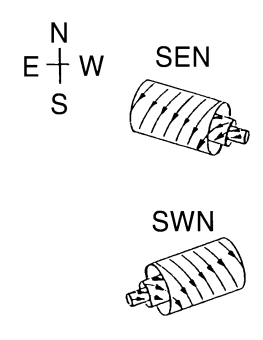

Fig. 18. Sketch showing the extended association between sunspots, filaments and MCs. The left panel shown the polarity of sunspots during two subsequent cycles. Note that the solar cycle number, $n$, is assumed to be an odd number. The middle panel shows the expected preferential magnetic structure of filaments in the two solar

Fig. 18 shows the magnetic polarities of sunspots in both solar hemispheres during two subsequent cycles. The top row of Fig. 18 shows the magnetic polarities for odd cycles, e.g., for cycle 21, when the Helios observations were taken. The bottom row shows the polarities during even cycles. The middle column sketches the magnetic structures for mid- to high-latitude filaments during the two cycles based on the hemispheric helicity rule found by Rust and Kumar (1994) who inferred that in filaments in the northern solar hemisphere the twist of the magnetic field lines is preferentially left-handed, whereas it is preferentially right-handed in the southern hemisphere. The right column of Fig. 18 finally presents the magnetic structure of MCs based on the interpretation of our observations (see also Bothmer and Schwenn, 1994).

Figure 18 implies that during odd cycles, e.g., during solar cycle 21 , filaments should preferentially evolve in association with SMEs into SN flux tubes (MCs) in the interplanetary medium, whereas in the following cycle preferentially NS flux tubes (MCs) should evolve, in agreement with our findings. Further evidence for the solar cycle dependence of MCs and their agreement with the magnetic structure of filaments based on the helicity rule has been recently presented by Bothmer and Rust (1997).

\section{Conclusions and summary}

Using the complete set of plasma and magnetic field data of the Helios $1 / 2$ spacecraft, we have identified 46 MCs between 0.3-1 AU during the period 1974-1981 hemispheres and the right panel shows the expected structure of MCs in interplanetary space. During odd $(n)$ cycles SN-MCs are dominant whereas NS-MCs dominate during even $(n+1)$ cycles (Bothmer and Rust, 1997)

between 0.3 and 1 AU. Additionally, we used solar wind data from the IMP, Pioneer and Voyager spacecraft for multi-spacecraft studies of MCs and to investigate the expansion of MCs in the outer heliosphere. Based on these investigations, we summarize the main properties of MCs in the heliosphere:

1. Our results support the close relationship between MCs and SMEs. $85 \%$ of the MCs observed by Helios $1 / 2$ were associated with a fast forward shock. Seven of the MCs were observed after a Helios-directed SME was detected by the Solwind-corongraph on the satellite P78/1. Without taking the duty cycle of the instruments into account, half of the MCs were found to be associated with passage of an IPC detected by the Helios-photometer as a white-light brightness enhancement in the interplanetary medium.

2. Between 0.3 and $1 \mathrm{AU}, \mathrm{MCs}$ have higher total plasma pressure and lower plasma- $\beta$ values $(\beta \ll 1)$ compared to the normal solar wind.

3. MCs can best be described as large-scale cylindrical magnetic flux tubes with observed longitudinal extents up to $\sim 60^{\circ}$. The axis of the flux tube commonly has a small inclination to the ecliptic, with its azimuthal direction being roughly directed near the EW-direction, i.e., normal to the sun-spacecraft line.

4. MCs can occur in four different magnetic configurations. The individual types are distinguished by the magnetic orientation at the center (the axis) of the $\mathrm{MC}$ and on its outer boundaries. The four configurations result from two possible directions of the field axis and two possible values of magnetic helicity.

5. MCs expand during their outward propagation. Their radial diameter, $s$, was found to increase between 0.3 
and 4.2 AU proportional to $R^{0.8}$. Consistent with this expansion, the plasma density inside MCs decreases, faster than the normal solar wind, proportional to $R^{-2.4}$ between 0.3 and $1 \mathrm{AU}$. According to this result, on average, an IPC type MC should be identifiable as a transient white-light brightness enhancement in the interplanetary medium up to a distance of $\sim 0.5 \mathrm{AU}$ from the Sun, whereas, without taking brightness enhancements into account that have been caused by compression due to the interaction of a MC with the ambient solar wind, a MC should at greater distances from the Sun lead to a transient white-light brightness depletion.

6. We found nine MCs identified in Helios and nearEarth solar wind data to be uniquely associated with quiescent filament disappearances (DBs) at mid to high solar latitudes outside active regions. In eight of the nine cases we found agreement between the magnetic flux tube structure of the MC and that of the associated filament as inferred from the orientation of the filament axis and its magnetic polarity, on the assumption of left-handed (right-handed) magnetic helicity dominance of filaments in the northern (southern) solar hemisphere. Further, the preferred east-west orientation of mid- to high-latitude quiescent prominences caused by differential rotation may explain the preference of MCs to have small axis inclinations to the ecliptic in the interplanetary medium. However, we note that some MCs may well be related to other solar activity features, such as flares. Also, other results do not necessarily imply that the filament itself evolves into the $\mathrm{MC}$ in interplanetary space. From a comparison of the spatial sizes of the features seen in $\mathrm{H} \alpha$-filtergrams and white-light coronagraph observations it seems likely that the filament may be either the bottom part of a large flux tube or just an indicator of the overlying coronal field structure.

7. There is a solar cycle dependence of the magnetic structure of MCs. Although MCs of opposite magnetic helicity occurred with equal frequency during 1974-1981, 3/4 of the MCs were associated with SN-rotations of the magnetic field vector, but only $\sim 1 / 4$ had NS-rotations. During the subsequent cycle predominately NS-rotations were found.

8. We suggest that the solar cycle variation of the magnetic structure of MCs can be explained by the magnetic field structure of disappearing filaments at the Sun and their solar cycle changes.

9. It is likely that magnetic reconnection occurs in the coronal loops overlying the prominence axis of a disappearing filament in association with a SME.

Acknowledgements. The authors thank both referees for their assistance in evaluating this paper. They further thank T.R. Sanderson for carefully reading the manuscript and D.F. Webb, N. Crooker, and H.V. Cane for helpful discussions. The authors also thank F.M. Neubauer for use of the Helios magnetic field data and J.F. Cooper for his assistance in using the $\mathrm{COHO}$ database at NSSDC. VB is grateful for the support given by the Max-Planck Insitut für Aeronomie, Katlenburg-Lindau, Germany during his $\mathrm{PhD}$ studies.
The Editor in chief thanks S. Kahler and another referee for their help in evaluating this paper.

\section{Appendix: minimum variance analysis for MCs}

During the passage of a MC, the magnetic field vector $B$ was measured at $N$ successive times $t$. The mean value of $B$ in Cartesian components is:

$$
\langle B\rangle=\frac{1}{N} \sum_{i=1}^{N} B^{i}
$$

with: $\quad B^{i}=\left(B_{x}^{i}, B_{y}^{i}, B_{z}^{i}\right), \quad i=1, \ldots, N$

A normal-vector $n$ for the direction of minimum variance of $B$ is to be determined. This can be achieved by calculating the mean quadratic deviation of the individual products $B^{i} \cdot n$ from $\left\langle B^{i}\right\rangle \cdot n$ :

$\sigma^{2}=\frac{1}{N} \sum_{i=1}^{N}\left(B^{i} \cdot n-\left\langle B^{i}\right\rangle \cdot n\right)^{2}$

Optimizing Eq. (A2) is equivalent to finding the smallest eigenvalue of the covariance-matrix $M_{\alpha, \beta}$ :

$M_{\alpha, \beta}=\left(\left\langle B_{\alpha} B_{\beta}\right\rangle-\left\langle B_{\alpha}\right\rangle\left\langle B_{\beta}\right\rangle\right)$

with: $\alpha, \beta \in\{x, y, z\}$

The individual $B_{\alpha, \beta}$ in Eq. (A3) are the Cartesian components of $B$ for a single measurement. The three calculated eigenvectors and eigenvalues of $M_{\alpha, \beta}$ correspond to the directions of minimum, intermediate and maximum variance of $B$, i.e. to the directions of a new principal axis (the minimum variance) system. The common nomenclature in the literature for the eigenvectors $\underline{e}_{i}$ and eigenvalues $\lambda_{i}$ is:

$-e_{1}, \lambda_{1}$ : eigenvector and eigenvalue for the direction of maximum variance

$-e_{2}, \lambda_{2}$ : eigenvector and eigenvalue for the direction of intermediate variance

$-e_{3}, \lambda_{3}$ : eigenvector and eigenvalue for the direction of minimum variance

The variance directions are well determined if the error criteria given by Lepping and Behannon (1980) and Siscoe and Suey (1972) are satisfied, i.e. if:

$$
\begin{aligned}
& \frac{\lambda_{2}}{\lambda_{3}} \geq 2 \\
& \varangle\left(B_{1}, B_{N}\right) \geq 30^{\circ}
\end{aligned}
$$

The approximate error of the minimum variance direction is $\sim 10^{\circ}$ (Burlaga and Behannon, 1982). The calculated variance directions in solar ecliptic coordinates are:

$$
\begin{aligned}
\varphi_{k} & =\operatorname{atan} \frac{y_{k}}{x_{k}} \\
\theta_{k} & =\operatorname{atan} \frac{\sqrt{x_{k}^{2}+y_{k}^{2}}}{z_{k}}
\end{aligned}
$$

with: $|e|=1 ; e_{k}=\left(x_{k}, y_{k}, z_{k}\right) ; k=1,2,3$ 
Finally, the measured components of $B$ in the minimum variance system are:

$B_{k}^{i} *=e_{k} \cdot B^{i}$

with:

$B_{1}^{*}=B_{x}^{*} \equiv$ component of maximum variance

$B_{2}^{*}=B_{y}^{*} \equiv$ component of intermediate variance

$B_{3}^{*}=B_{z}^{*} \equiv$ component of minimum variance

\section{References}

Bame, S. J., J. R. Asbridge, W. C. Feldman, J. T. Gosling, and R. D. Zwick, Bi-directional streaming of solar wind electrons $>80 \mathrm{eV}$; ISEE evidence for a closed field structure within the driver gas of an interplanetary shock, Geophys. Res. Lett., 8, 173-176, 1981.

Borrini, G., J. T. Gosling, S. J. Bame, W. C. Feldman, Analysis of shock wave disturbances observed at 1 AU from 1971 through 1978, J. Geophys. Res., 87, 4365-4373, 1982.

Bothmer, V., Die Struktur magnetischer Wolken im Sonnenwind -Zusammenhang mit eruptiven Protuberanzen und Einflu $\beta$ auf die Magnetosphäre der Erde, PhD Thesis, University Göttingen, 1993.

Bothmer, V., and D. M. Rust, The field configuration of magnetic clouds and the solar cycle, in Proc. of $A G U$ Conf. on Coronal Mass Ejections: Causes and Consequences, Geophys. Monogr. Series, in press, 1997.

Bothmer, V., and R. Schwenn, Magnetic structures at sector boundaries in the inner heliosphere, in Solar Wind Seven, Ed. E. Marsch and R. Schwenn, Pergamon, Oxford, pp. 51-154, 1992a.

Bothmer, V., and R. Schwenn, Magnetic cloud observations by the Helios spacecraft, in Solar Wind Seven, Ed. E. Marsch and R. Schwenn, Pergamon, Oxford, pp. 599-602, 1992b.

Bothmer, V., and R. Schwenn, Eruptive prominences as sources of magnetic clouds in the solar wind, Proc of the II Soho Workshop at Elba, Italy, 1993, Space Sci. Rev., 70, 215-220, 1994.

Bothmer, V., and R. Schwenn, The interplanetary and solar causes of major geomagnetic storms. J. Geomagn. Geoelectr., 47, 11271132, 1995.

Bothmer, V., and R. Schwenn, Signatures of fast CMEs in interplanetary space, Adv. Space Res., 17, 319-322, 1996.

Bothmer, V., R. G. Marsden, T. R. Sanderson, K. J. Trattner, K.-P. Wenzel, A. Balogh, R.J. Forsyth, and B.E. Goldstein, The Ulysses south polar pass: transient fluxes of energetic ions, Geophys. Res. Lett., 3369-3372, 1995.

Bothmer, V., M. I. Desai, R. G. Marsden, T. R. Sanderson, K. J. Trattner, K.-P. Wenzel, J. T. Gosling, A. Balogh, R. J. Forsyth, and B.E. Goldstein, Ulysses observations of open and closed magnetic field lines within a coronal mass ejection, Astron, Astrophys., 316, 493-498, 1996a.

Bothmer, V., R. G. Marsden, T. R. Sanderson, K. J. Trattner, K.-P. Wenzel, A. Balogh, R. J. Forsyth, B. E. Goldstein, Y. Uchida, and H. S. Hudson, Energetic particles and coronal mass ejections in the high latitude heliosphere: Ulysses-LET observations, Solar Wind Eight, AIP conf. Proc., 382, Woodbury, New York, 445-448, 1996b.

Burlaga, L. F., Magnetic clouds and force-free fields with constant alpha, J. Geophys, Res., 93, 7217-7224, 1988.

Burlaga, L. F., Magnetic clouds, in Physics of the Inner Heliosphere vol. II, Ed. R. Schwenn and E. Marsch, Springer-Verlag, Berlin, Heidelberg, pp. 1-22, 1991.

Burlaga, L. F., and K. W. Behannon, Magnetic clouds: Voyager observations between 2 and 4 AU, Sol. Phys, 81, 181, 1982

Burlaga, L. F., E. Sittler, F. Mariani, and R. Schwenn, Magnetic loop behind an interplanetary shock: Voyager, Helios, and IMP 8 observations, J. Geophys. Res., 86, 6673-6684, 1981.
Burlaga, L. F., L. Klein, N. R. Sheeley Jr., D. J. Micheles, R. A. Howard, M. J. Koomen, R. Schwenn, and H. Rosenbauer, A magnetic cloud and a coronal mass ejection, Geophys. Res. Lett., 9, 1317-1320, 1982.

Burlaga, L. F., K. W. Behannon, and L. W. Klein, Compound streams, magnetic clouds, and major geomagnetic storms, J. Geophys. Res., 92, 5725-5734, 1987.

Burlaga, L. F., R. P. Lepping, and J. A. Jones, Global configuration of a magnetic cloud, in Physics of Magnetic Flux Ropes, Eds. E.R. Priest, L.C. Lee, C.T. Russell, AGU Geophysical Monograph, 58, 373-377, 1990.

Burlaga, L. F., K. W. Behannon, and L. W. Klein, Compound streams, magnetic clouds, and major geomagnetic storms, J. Geophys. Res., 92, 5725-5734, 1987.

Burlaga, L. F., L. Klein, N. R. Sheeley Jr., D. J. Michels, R. A. Howard, M. J. Koomen, R. Schwenn, and H. Rosenbauer, A magnetic cloud and a coronal mass ejection, Geophys. Res. Lett., 9, 1317-1320, 1982.

Burlaga, L. F., E. Sittler, F. Mariani, and R. Schwenn, Magnetic loop behind an interplanetary shock: Voyager, Helios, and IMP 8 observations, J. Geophys. Res., 86, 6673-6684, 1981.

Cane, H. V., S. W. Kahler, and N. R. Sheeley, Jr., Interplanetary shocks preceded by solar filament eruptions, J. Geophys, Res., 91, 13321-13329, 1986.

Chen, J., Theory of prominence eruption and propagation: interplanetary consequences, J. Geophys. Res., 101, 2749927519, 1996

Farrugia, C. J., V. A. Osherovich, and L. F. Burlaga, Magnetic flux rope versus the spheromak as models for interplanetary magnetic clouds, J. Geophys. Res., 100, 12293-12306, 1995.

Farrugia, C. J., L. F. Burlaga, V. A. Osherovich, L. G. Richardson, M. P. Freeman, R. P. Lepping, and A. J. Lazarus, A study of an expanding interplanetary magnetic cloud and its interaction with the Earth's magnetosphere: the interplanetary aspect, J. Geophys. Res., 98, 7621-7632, 1993.

Feynman, J., and S. F. Martin, The initiation of coronal mass ejections by newly emerging magnetic flux, J. Geophys. Res., 100, 3355-3367, 1995.

Goldstein, H., On the field configuration in magnetic clouds, in Solar Wind Five, NASA Conf. Publ., CP-2280, 731-733, 1983.

Gosling, J. T., The solar flare myth, J. Geophys. Res., 98, 1893718949, 1993a.

Gosling, J. T., Coronal mass ejections: the link between solar and geomagnetic activity, Phys. Fluids., B5, 2638-2645, 1993 b.

Gosling, J. T., Coronal mass ejections and magnetic flux ropes in interplanetary space, in Physics of Magnetic Flux Ropes, Eds. E.R. Priest, L.C. Lee, and C.T. Russell, $A G U$ Geophysical Monograph, 58, 343-364, 1990.

Gosling, J. T., and D. J. McComas, Field line draping about fast coronal mass ejecta: a source of strong out-of the ecliptic interplanetary magnetic fields, Geophys. Res. Lett., 14, 355-358, 1987.

Gosling, J. T., V. Pizzo, and S. J. Bame, Anomalously low proton temperatures in the solar wind following interplanetary shock waves: evidence for magnetic bottles?, J. Geophys. Res., 78, 2001, 1973.

Gosling, J. T., D.N. Baker, S. J. Bame, W. C. Feldman, R. D. Zwickl, and E. J. Smith, Bidirectional solar wind electron heat flux events, J. Geophys. Res., 92, 8519-8535, 1987.

Gosling, J. T., D. J. McComas, and J. L. Phillips, Counterstreaming solar wind halo electron events on open field lines?, in Solar Wind Seven, Ed. E. Marsch and R. Schwenn, Pergamon, Oxford, pp. 619-622, 1992.

Gosling, J. T., D. J. McComas, J. L. Phillips, L. A. Weiss, V. J. Pizzo, B. E. Goldstein, and R. J. Forsyth, A new class of forward-reverse shock pairs in the solar wind, Geophys. Res. Lett., 21, 2271-2274, 1994a.

Gosling, J. T., S. J. Bame, D. J. McComas, J. L. Phillips, E. E. Scime, V. J. Pizzo, B. E. Goldstein and A. Balogh, A forwardreverse shock pair in the solar wind driven by over-expansion of 
a coronal mass ejection: Ulysses, Geophys. Res. Lett., 21, 22712274, 1994b.

Gosling, J. T., S. J. Bame, W. C. Feldman, D. J. McComas, J. L. Phillips, B. Goldstein, M. Neugebauer, J. Burkepile, A.J. Hndhusen, and L. Acton, The band of solar wind variability at low heliographic latitudes near solar activity minimum: plasma results from the Ulysses rapid latitude scan, Geophys, Res. Lett., 22, 1753-1756, 1995a.

Gosling, J. T., S. J. Bame, D. J. McComas, J. L. Phillips, A. Balogh, and K. T. Strong, Coronal mass ejections at high heliographic latitudes: Ulysses, Space Sci. Rev., 72, 133-136, $1995 \mathrm{~b}$

Gosling, J. T., J. Birn, and M. Hesse, Three-dimensional magnetic reconnection and the magnetic topology of coronal mass ejection events, Geophys. Res. Lett., 22, 869-872, 1995c.

Harrison, R. A., Solar coronal mass ejections and flares, Astron. Astrophys., 162, 283-291, 1986.

Hoeksema, J. T., and P. H. Scherrer, The solar magnetic field 1976 through 1985, RPP-UAG-94, Natl. Geophys. Data Center, Boulder, Co., 1986.

Hundhausen, A. J., Coronal expansion and solar wind, physics and chemistry in space, Springer-Verlag, Berlin Heidelberg, New York, 1972.

Hundhausen, A. J., J. T. Burkepile, and O. C. St. Cyr, Speeds of coronal mass ejections: SMM observations from 1980 and 1984-1989, J. Geophys. Res., 99, 6543-6552, 1994.

Jackson, B. V., D. F. Webb, P. L. Hick, and J. L. Nelson, Catalog of Helios $90^{\circ}$ photometer events, Phillips Laboratory, Directorate of Geophysics, Hanscom Air Force Base, MA 017313010, USA, Sci. Rep. 4, 1994

Kahler, S. W. and D. V. Reames, Probing the magnetic topologies of magnetic clouds by means of solar energetic particles, J. Geophys. Res., 96, 9419-9424, 1991.

King, J. H., Long-term solar wind variations and associated data sources, J. Geomag. Geolelectr., 43, 865-880, 1991.

Klein, L. W., and L. F. Burlaga, Interplanetary magnetic clouds at 1 AU, J. Geophys. Res., 87, 613-624, 1982.

Kumar, A., and D. M. Rust, Interplanetary magnetic clouds, helicity conservation, and intrinsic-scale flux-ropes, J. Geophys. Res., 101, 15667-15684, 1996.

Lemen, J. R., L. W. Acton, D. Alexander, A. B. Galvin, K. L. Harvey, J. T. Hoeksema, X. Zhao, and H. S. Hudson, Solar identification of solar-wind distrubances observed at Ulysses, Solar Wind Eight, AIP Conf. Proc., 382, Woodbury, New York, 92-95, 1996.

Lepping, R. P., and K. W. Behannon, Magnetic field directional discontinuities: 1. minimum variance errors, J. Geophys. Res., 85, 4695-4703, 1980.

Lepping, R. P., J. A. Jones, and J. F. Burlaga, Magnetic field structure of interplanetary magnetic clouds at $1 \mathrm{AU}$, J. Geophys. Res., 95, 11957-11965, 1990.

Lüst, R., and A. Schlüter, Kraftfreie Magnetfelder, Z. Astrophys., 34, 263-282, 1954.

Martin, S. F., and A. H. McAllister, Predicting the sign of helicity in erupting filaments and coronal mass ejections, in Proc. of AGU conf. on Coronal Mass Ejections: Causes and Consequences, Geophys. Monogr. Series, in press, 1997.

Martin, S. F., R. Bilimoria, and P. W. Tracadas, Magnetic field configurations basic to filament channels, in Solar Surface Magnetism, Ed. R.J. Rutten and C.J. Schrijver, SpringerVerlag, Berlin Heidelberg, New York, pp. 303-338, 1994.

Marubashi, K., Structure of the interplanetary magnetic clouds and their solar origins, Adv. Space Res., 6, 335-338, 1986.

McComas, D. J., J. T., Gosling, D. Winterhalter, and E. J. Smith, Interplanetary magnetic field draping about fast coronal mass ejecta in the outer heliosphere, J. Geophys, Res., 93, 2519-2526, 1988.

Montgomery, M. D., J. R. Asbridge, S. J. Bame, and W. C. Feldman, Solar wind electron temperature depressions following some interplanetary shock waves: evidence for magnetic merging? J. Geophys. Res., 79, 3103, 1974.
Musmann, G., F. M. Neubauer, A. Maier, and E. Lammers, Das Förstersonden-Magetfeldexperiment (E2), Raumfahrtforschung, 19/5, 232-237, 1975.

Nakagawa, T., A. Nishida, T. Saito, Planar magnetic structures in the solar wind, J. Geophys. Res., 94, 11761-11775, 1989.

Neugebauer, M., D. R. Clay, J. T. Gosling, The origins of planar magnetic structures in the solar wind, J. Geophys. Res., 98, 9383, 1993.

Phillips, L. J., J. T. Gosling, D. J. McComas, S. J. Bame, and W. C. Feldman, Quantitative analysis of bidirectional electron fluxes within coronal mass ejections at $1 \mathrm{AU}$, in Solar Wind Seven, Ed. E. Marsch and R. Schwenn, Pergamon, Oxford, pp. 651-656, 1992.

Priest, E. R., The equilibrium of magnetic flux ropes, $A G U$ Geophys. Mongraph, 58, 1, 1990.

Priest, E. R., Ed., Dynamics and Structure of Quiescent Solar Prominences, Astrophysics and Space Science Library, Kluwer Academic Publishers, 101 Phillip Drive, Norwell, MA 02061 USA, 1989.

Priest, E. R., A. A. van Ballegooijen, and D. H. MacKay, A model for dextral and sinistral prominences, Ap. J., 460, 530-543, 1996.

Priest, E. R., The initiation of solar coronal mass ejections by magnetic nonequilibrium, Astron. Astrophys., 328, 848-855, 1988.

Richardson, R. S., Solar hydrogen vortices, Astrophys. J., 93, 24 28., 1941.

Richardson, I. G., and H. V. Cane, Signatures of shock drivers in the solar wind and their dependence on the solar source location, J. Geophys. Res., 98, 15295-15304, 1993.

Rosenbauer, H., R. Schwenn, H. Miggenrieder, B. Meyer, H. Grünwaldt, K.-H. Mühlhäuser, H. Pelkofer, and J. H. Wolfe, Die Instrumente des Plasmaexperimentes auf den HELIOS-Sonnensonden, Luft-und Raumfahrt, Weltraumforschung/Weltraumtechnologie, BMFT-FB, W81-015, 1981.

Rust, D.M., Spawning and shedding helical magnetic fields in the solar atmosphere, Geophys. Res. Lett., 21, 241-244, 1994.

Rust, D.M., Magnetic fields in quiescent solar prominences I. observations, Astrophys. J., 150, 313, 1967.

Rust, D.M., and A. Kumar, Helical charging and eruption of magnetic flux from the Sun, Proc. Third SOHO Workshop, ESA SP-373, 39-43, 1994a.

Rust, D. M., and A. Kumar, Helical magnetic fields in filaments, Solar Phys., 155, 69-98, 1994b.

Schmidt, W.K.H., and V. Bothmer, Stereoscopic viewing of solar coronal and interplanetary activity, Adv. Space Res., 17, 369376, 1996

Schwenn, R., Large-scale structure of the interplanetary medium, in Physics of the the Inner Heliosphere, vol. I, Ed. R. Schwenn and E. Marsch, Springer-Verlag, Berlin Heidelberg, New York, 1990.

Schwenn, R., Relationship of coronal transients to interplanetary shocks: 3 D aspects, Space Sci. Rev., 44, 139-168, 1986.

Schwenn, R., H. Rosenbauer, and K.-H. Mühlhäuser, Singly-ionized helium in the driver gas of an interplanetary shock wave, Geophys. Res. Lett., 7, 201-204, 1980.

Schwenn, R., H. Rosenbauer, and H. Miggenrieder, Das Plasmaexperiment auf HELIOS (E1), Raumfahrtforschung, 19/5, 226231, 1975.

Sheeley, N.R. Jr., R.A. Howard, M.J. Koomen, D.J. Michles, R. Schwenn, K.-H. Mühlhäuser, and H. Rosenbauer, Coronal mass ejections and interplanetary shocks, J. Geophys. Res., 90, 163$175,1985$.

Siscoe, G.L., and R.W. Suey, Significance criteria for variance matrix applications, J. Geophys. Res., 77, 1321-1322, 1972.

Smith, C.W., and J.L. Phillips, The role of coronal mass ejections and interplanetary shocks in interplanetary magnetic field statistics and solar magnetic flux ejection, J. Geophys. Res., 102, 249-261, 1997.

Sonnerup, B.U.Ö., and L.J. Cahill, Magnetopause structure and attitude from Explorer 12 observations, J. Geophys. Res., 72, 171-183, 1967. 
Tsurutani, B.T., W.D. Gonzalez, F. Tang, and Y.T. Lee, Great magnetic stroms, Geophys. Res. Lett., 19, 73-76, 1992.

van Ballegooijen, A. A., and P.C.H. Martens, Magnetic fields in quiescent prominences, Astrophys. J., 361, 283-289, 1990.

Vandas, M., and S. Fischer, Spherical and cylindrical models of magnetized plasma clouds and their comparison with spacecraft data, Planet. Space Sci., 39, 1147-1154, 1991.

Vandas M., S. Fischer, P. Pelant, and A. Geranios, Evidence for spheroidal structure of magnetic clouds, J. Geophys. Res., 98, 21061-21069, 1993.

Webb, D.F., Erupting prominences and the geometry of coronal mass ejections, J. Geophys. Res., 93, 1749-1758, 1988.

Webb, D.F. and B.V. Jackson, The identification and characteristics of solar mass ejections observed in the heliosphere by the Helios 2 photometers, J. Geophys. Res., 95, 20641-20661, 1990.

Webb, D.F., and A.J. Hundhausen, Activity associated with the solar origin of coronal mass ejections, Sol. Phys., 108, 383-401, 1987.

Webb, D., B. Jackson, P. Hick, R. Schwenn, V. Bothmer, and D. Reames, Comparison of CMEs, magnetic fields, and bidirectionally streaming proton events in the heliosphere using Helios data, Adv. Space Res., 13, 9, 971-974, 1993.
Weiss, L.A., J.T. Gosling, A.H. McAllister, A.J. Hundhausen, J.T. Burkepile, J.L. Phillips, K.T. Strong, and R.J. Forsyth, A comparison of interplanetary coronal mass ejections at Ulysses with Yohkoh soft x-ray coronal events, Astron. Astrophys., 316, 384-395, 1996.

Wilson, R.M., Geomagnetic response to magnetic clouds, Planet. Space Sci., 35, 3, 329-335, 1987.

Wilson, R.M. and E. Hildner, On the association of magnetic clouds with disappearing filaments, J. Geophys. Res., 91, 58675872, 1986.

Wilson, R.M., and E. Hildner, Are interplanetary magnetic clouds manifestations of coronal transients at 1 AU? Sol. Phys., 91, 169-180, 1984.

Wright, C.S., Catalog of solar filament disappearances 1964-1980, Rep. UAG-100, Natl. Geophys. Data Center, Boulder, Co., 1991.

Yang, H.-S., Q.F. Hong, and Y.J. Ding, A constant- $\alpha$ force-freefield analysis of the active region AR 4711 of February, 1986, Sol. Phys., 117, 57-68, 1988.

Zhang, G., and L.F. Burlaga, Magnetic clouds, geomagnetic disturbances, and cosmic ray decreases, J. Geophys. Res., 93, 2511-2518, 1988. 